\title{
Stability of radially symmetric travelling waves in reaction-diffusion equations
}

\section{Stabilité des ondes progressives à symétrie sphérique dans les équations de réaction-diffusion}

\author{
Violaine Roussier \\ Département de mathématique, Université de Paris-Sud, bât 425, 91405 Orsay cedex, France \\ Received 21 October 2002; accepted 15 April 2003
}

Available online 4 October 2003

\begin{abstract}
The asymptotic behaviour as $t$ goes to infinity of solutions $u(x, t)$ of the multidimensional parabolic equation $u_{t}=\Delta u+F(u)$ is studied in the "bistable" case. More precisely, we consider the stability of spherically symmetric travelling waves with respect to small perturbations. First, we show that such waves are stable against spherically symmetric perturbations, and that the perturbations decay like $(\log t) / t^{2}$ as $t$ goes to infinity. Next, we observe that this stability result cannot hold for arbitrary (i.e., non-symmetric) perturbations. Indeed, we prove that there exist small perturbations such that the solution $u(x, t)$ does not converge to a spherically symmetric profile as $t$ goes to infinity. More precisely, for any direction $k \in S^{n-1}$, the restriction of $u(x, t)$ to the ray $\{x=k r \mid r \geqslant 0\}$ converges to a $k$-dependent translate of the one-dimensional travelling wave.

(C) 2004 L'Association Publications de l'Institut Henri Poincaré. Published by Elsevier B.V. All rights reserved
\end{abstract}

\section{Résumé}

On étudie le comportement pour les grands temps des solutions $u(x, t)$ de l'équation parabolique $u_{t}=\Delta u+F(u)$ dans le cas "bistable" et dans tout l'espace, en dimension supérieure. Plus précisément, on s'intéresse à la stabilité d'ondes progressives à symétrie sphérique pour de petites perturbations. Dans un premier temps, on montre que cette famille d'ondes est stable pour des perturbations à symétrie sphérique et que cette perturbation décroît comme $(\log t) / t^{2}$ quand $t$ tend vers l'infini. On montre ensuite que cette stabilité est mise en défaut pour des perturbations quelconques. En effet, on met en évidence des perturbations pour lesquelles la solution ne tend pas vers une onde à symétrie sphérique : dans chaque direction $k \in S^{n-1}$, la restriction de $u(x, t)$ au rayon $\{x=k r, r \geqslant 0\}$ converge vers un translaté de l'onde progressive unidimensionnelle dépendant de $k$. (C) 2004 L'Association Publications de l'Institut Henri Poincaré. Published by Elsevier B.V. All rights reserved

MSC: 35B40; 35K57; 35B 35

Keywords: Semilinear parabolic equation; Long-time asymptotics; Stability; Travelling waves

E-mail address: violaine.roussier@math.u-psud.fr (V. Roussier).

0294-1449/\$ - see front matter (C) 2004 L'Association Publications de l'Institut Henri Poincaré. Published by Elsevier B.V. All rights reserved doi:10.1016/j.anihpc.2003.04.002 


\section{Introduction}

We consider the initial value problem for the semilinear parabolic equation

$$
\begin{cases}u_{t}(x, t)=\Delta u(x, t)+F(u(x, t)), & x \in \mathbf{R}^{n}, t>0, \\ u(x, 0)=u_{0}(x), & x \in \mathbf{R}^{n},\end{cases}
$$

where $u \in \mathbf{R}$ and $n \geqslant 2$. Throughout this paper, it is assumed that the nonlinearity $F$ is a continuously differentiable function on $\mathbf{R}$ satisfying the following assumptions:

(i) $F(0)=F(1)=0$;

(ii) $F^{\prime}(0)<0, F^{\prime}(1)<0$;

(iii) There exists $\mu \in(0,1)$ such that $F(u)<0$ if $u \in(0, \mu)$ and $F(u)>0$ if $u \in(\mu, 1)$;

(iv) $\int_{0}^{1} F(u) d u>0$.

A typical example is the cubic nonlinearity

$$
F(u)=2 u(1-u)(u-\mu) \quad \text { where } 0<\mu<1 / 2 .
$$

Eq. (1) is a classical model for spreading and interacting particles, which has been often used in biology (population dynamics, propagation of nerves pulses), in physics (shock waves), or in chemistry (chemical reactions, flame propagation). Fisher [5] first proposed a genetical context in which the spread of advantageous genetical traits in a population was modeled by Eq. (1). At the same time, Kolmogorov, Petrovskii and Piskunov [11] gave a mathematical treatment of this equation for a slightly different nonlinearity. Later on, Aronson and Weinberger [1] also discussed the genetical background in some details. In their terminology, the nonlinearity satisfying (i) to (iv) is referred to as the "heterozygote inferior" case. In mathematical terms, this is called the "bistable" case as, by (i) and (ii), $u \equiv 0$ and $u \equiv 1$ are both stable steady states.

As far as the initial value problem is concerned, if $u_{0}$ is a continuous function from $\mathbf{R}^{n}$ to $(0,1)$ which goes to 0 as $|x|$ goes to infinity, then there exists a unique solution $u(x, t)$ of Eq. (1) with the same properties as $u_{0}$ for any $t \geqslant 0$.

One question of interest for this reaction-diffusion equation is the behaviour, as $t$ goes to infinity, of the solutions $u(x, t)$ of (1). In one space dimension, a prominent role is played by a family of particular solutions of (1), called travelling waves. These are uniformly translating solutions of the form

$$
u(x, t)=w_{0}(x-c t),
$$

where $c \in \mathbf{R}$ is the speed of the wave. The profile $w_{0}$ satisfies the ordinary differential equation:

$$
w_{0}^{\prime \prime}+c w_{0}^{\prime}+F\left(w_{0}\right)=0, \quad x \in \mathbf{R},
$$

together with the boundary conditions at infinity

$$
\lim _{x \rightarrow-\infty} w_{0}(x)=1 \quad \text { and } \quad \lim _{x \rightarrow+\infty} w_{0}(x)=0 .
$$

These waves are characterized by their time independent profile and usually represent the transport of information in the above models. They also often describe the long-time behaviour of many solutions.

Since Fisher and KPP, there has been an extensive literature on the subject. In the one dimensional bistable case, Kanel [9] proved that there exist a unique speed $c>0$ and a unique (up to translations) monotone profile $w_{0}$, satisfying (3), (4). Moreover, $\left|w_{0}\right|$ (resp. $\left|1-w_{0}\right|$ ) decays exponentially fast as $x$ goes to $+\infty$ (resp. $-\infty$ ). From now on, we fix $w_{0}$ by choosing $w_{0}(0)=1 / 2$. For example, if $F$ is given by $(2)$, one finds $c=1-2 \mu \in(0,1)$ and $w_{0}(x)=\left(1+\mathrm{e}^{x}\right)^{-1}$. 
Afterwards, Sattinger [14] was interested in the local stability of travelling waves. He proved that the family $\left\{w_{0}(\cdot-\gamma), \gamma \in \mathbf{R}\right\}$ is normally attracting. More precisely, for any initial data $u_{0}$ of the form

$$
u_{0}(x)=w_{0}(x)+\varepsilon v_{0}(x),
$$

where $\varepsilon>0$ is sufficiently small and $v_{0}$ bounded in a weighted space, Sattinger proved that there exist a $\mathcal{C}^{1}$ function $\rho(\varepsilon)$ and positive constants $K$ and $\gamma$ such that the solution $u(x, t)$ of (1) satisfies

$$
\left\|u(x+c t, t)-w_{0}(x+\rho(\varepsilon))\right\| \leqslant K \mathrm{e}^{-\gamma t}, \quad t \geqslant 0,
$$

in an appropriate weighted norm. This is the local stability of travelling waves in one dimension. Sattinger's proof uses the spectral properties of the linearised operator $L_{0}=\partial_{y}^{2}+c \partial_{y}+F^{\prime}\left(w_{0}\right)$ around the travelling wave $w_{0}$ in the $c$-moving frame. These properties can be summarized as follows:

Let $\phi_{0}=\bar{\alpha} w_{0}^{\prime}$ and $\psi_{0}=\mathrm{e}^{c x} \phi_{0}$ where $\bar{\alpha}>0$ is chosen so that

$$
\int_{\mathbf{R}} \phi_{0}(x) \psi_{0}(x) \mathrm{d} x=1 .
$$

Then, $\phi_{0}$ is an eigenfunction of $L_{0}$ (associated with the eigenvalue 0 ), and $\psi_{0}$ is the corresponding eigenfunction of the adjoint operator $L_{0}^{*}$ :

$$
\begin{aligned}
& \phi_{0}^{\prime \prime}+c \phi_{0}^{\prime}+F^{\prime}\left(w_{0}\right) \phi_{0}=0, \\
& \psi_{0}^{\prime \prime}-c \psi_{0}^{\prime}+F^{\prime}\left(w_{0}\right) \psi_{0}=0 .
\end{aligned}
$$

Moreover, there exists some $\gamma>0$ such that the spectrum of $L_{0}$ in $L^{2}(\mathbf{R})$ is included in $\left.]-\infty,-\gamma\right] \cup\{0\}$, see [6,14]. Since the eigenvalue 0 is isolated, there exists a projection operator $P$ onto the null space of $L_{0}$. This operator is given by

$$
P u=\frac{1}{2 \pi \mathrm{i}} \int_{\Gamma} R\left(\lambda, L_{0}\right) u \mathrm{~d} \lambda
$$

where $R\left(\lambda, L_{0}\right)=\left(\lambda-L_{0}\right)^{-1}$ and $\Gamma$ is a simple closed curve in the complex plane enclosing the eigenvalue 0 , see $[14,15]$. Define the complementary spectral projection $Q=I-P$ where $I$ is the identity operator in $L^{2}(\mathbf{R})$. These projection operators $P$ and $Q$ are also given by

$$
P u=\left(\int_{\mathbf{R}} u(x) \psi_{0}(x) \mathrm{d} x\right) \phi_{0}, \quad Q u=(I-P) u,
$$

see for instance [10,14]. The spectral subspace corresponding to the eigenvalue 0 is defined by $\left\{u \in L^{2}(\mathbf{R}) \mid u=\right.$ $P u\}$ and its supplementary by

$$
\mathcal{R}=\left\{u \in L^{2}(\mathbf{R}) \mid u=Q u\right\}=\left\{u \in L^{2}(\mathbf{R}) \mid P u=0\right\} .
$$

Then $\mathcal{R}$, equipped with the $L^{2}$ norm, is a Banach space and $\left.L_{0}\right|_{\mathcal{R}}$ generates an analytic semi-group which satisfies $\left\|\mathrm{e}^{t L_{0}}\right\|_{\mathcal{L}(\mathcal{R})} \leqslant c_{0} \mathrm{e}^{-\gamma t}$ for all $t \geqslant 0$.

On the other hand, Fife and McLeod [4] proved the global stability of travelling waves: they showed, using comparison theorems, that if $u_{0}$ satisfies $0 \leqslant u_{0} \leqslant 1$ and $\liminf _{-\infty} u_{0}(x)>\mu, \limsup _{+\infty} u_{0}(x)<\mu$, then the solution $u(x, t)$ of (1) approaches exponentially fast in time a translate of the travelling wave in the supremum norm. Fife [3] also highlighted other possible types of asymptotic behaviour: if $u_{0}$ vanishes at infinity in $x$ and if the solution converges uniformly to 1 on compact sets, then $u(x, t)$ behaves as a pair of diverging fronts where a wave goes off in each direction.

In higher dimensions, Aronson and Weinberger [2], Xin and Levermore [17,12] and Kapitula [10] were interested in planar travelling waves. These are particular solutions of equation (1) of the form $u(x, t)=w_{0}(x$. 
$k-c t$ ) where $k \in S^{n-1}$. Existence of such solutions can be proved as in the one-dimensional case, but the stability analysis is quite different: unlike in the one-dimensional case, the gap in the spectrum of the linearised operator around the travelling wave disappears. Instead, there exists continuous spectrum all the way up to zero which is due, intuitively, to the effects of the transverse diffusion. To overcome this difficulty, Kapitula decomposed the solution $u(x, t)$ as

$$
u(x, t)=w_{0}(x \cdot k-c t+\rho(x, t))+v(x, t),
$$

where $\rho(x, t)$ represents a local shift of the travelling wave and $v(x, t)$ a transverse perturbation in $\mathcal{R}$. The equation for $\rho$ can be analyzed by the one-dimensional result and Fourier transform, while the transverse perturbation $v$ satisfies a semilinear heat equation in $\mathbf{R}^{n-1}$. Therefore, Kapitula proved that the perturbation decays to zero with a rate of $\mathrm{O}\left(t^{-(n-1) / 4}\right)$ in $H^{k}\left(\mathbf{R}^{n}\right), k \geqslant[(n+1) / 2]$.

Apart from this particular planar case, Aronson and Weinberger [2] also studied the asymptotic behaviour of other solutions in higher dimensions. They proved that the state $u \equiv 0$ is stable with respect to perturbations which are not too large on too large a set, but is unstable with respect to some perturbations with bounded support. Moreover, assuming $u_{0}$ vanishes at infinity in $x$ and $u$ converges to 1 as $t$ goes to infinity, they showed that the disturbance is propagated with asymptotic speed $c$.

Finally, Uchiyama [16] and Jones [7] were interested in spherically symmetric solutions. If $u_{0}$ is spherically symmetric with $\lim \sup _{|x| \rightarrow+\infty} u_{0}(x)<\mu$, and if the solution $u(x, t)$ of (1) with initial data $u_{0}$ converges to 1 uniformly on compact sets as $t$ goes to infinity, they proved that there exists a function $g(t)$ such that

$$
\lim _{t \rightarrow+\infty} \sup _{x \in \mathbf{R}^{n}}\left|u(x, t)-w_{0}(|x|-c t+g(t))\right|=0 .
$$

Jones proved with dynamical systems considerations that $\lim _{t \rightarrow+\infty} g(t) / t=0$ and Uchiyama precised, using energy methods and comparison theorems, that there exists some $L \in \mathbf{R}$ such that

$$
\lim _{t \rightarrow+\infty}\left(g(t)-\frac{n-1}{c} \log t\right)=L .
$$

This important result establishes the existence of a family of asymptotic solutions of (1), which we call spherically symmetric travelling waves: $W(x, t)=w_{0}\left(|x|-c t+\frac{n-1}{c} \log t\right)$ and its translates in time. It also shows that this family is asymptotically stable with respect to spherically symmetric perturbations.

We give in the first section of this paper another method, based on Kapitula's decomposition, which enables us to get more information on how fast the solution $u(x, t)$ of (1) converges to a travelling wave and on the asymptotic behaviour of the function $g(t)$. To do that, we introduce the following Banach spaces:

$$
\begin{aligned}
& Y=H^{1}\left(\mathbf{R}^{+}\right), \\
& X=\left\{u: \mathbf{R}^{n} \rightarrow \mathbf{R} \mid \exists \tilde{u} \in Y \text { so that } u(x)=\tilde{u}(|x|) \text { for } x \in \mathbf{R}^{n}\right\} \\
& \|u\|_{X}=\|\tilde{u}\|_{Y}=\left(\int_{0}^{\infty}|\tilde{u}(r)|^{2}+\left|\tilde{u}_{r}(r)\right|^{2} \mathrm{~d} r\right)^{1 / 2} .
\end{aligned}
$$

Note that $X$ is included in $H^{1}\left(\mathbf{R}^{n}\right) \cap L^{\infty}\left(\mathbf{R}^{n}\right)$ and contains spherically symmetric functions. Then, we prove in the first section the following theorem:

Theorem 1. Assume $F$ is a "bistable" non-linearity. There exist positive constants $R_{0}, \delta_{0}, c_{1}, c_{2}, \gamma_{0}$ such that, if $u_{0}: \mathbf{R}^{n} \rightarrow \mathbf{R}$ is a spherically symmetric function satisfying

$$
\left\|u_{0}(x)-w_{0}(|x|-R)\right\|_{X} \leqslant \delta
$$


for some $R \geqslant R_{0}$ and some $\delta \leqslant \delta_{0}$, then Eq. (1) has a unique solution $u \in C^{0}\left([0,+\infty)\right.$, X) with initial data $u_{0}$. Moreover, there exists $\rho \in C^{1}([0,+\infty))$ such that

$$
\left\|u(x, t)-w_{0}(|x|-s(t))\right\|_{X}+\left|\rho^{\prime}(t)\right| \leqslant c_{1} \delta \mathrm{e}^{-\gamma_{0} t}+c_{2} \frac{\log (R+c t)}{(R+c t)^{2}}
$$

for all $t \geqslant 0$, where

$$
s(t)=R+c t-\frac{n-1}{c} \log \left(\frac{R+c t}{R}\right)+\rho(t) .
$$

This first theorem shows that the family of spherically symmetric travelling waves is asymptotically stable for small symmetric perturbations. Indeed, any small perturbation tends to zero with a rate of $\mathrm{O}\left(\log t / t^{2}\right)$. Moreover, as $\left|\rho^{\prime}(t)\right|$ is bounded by an integrable function of time, the function $\rho(t)$ converges to a constant $\rho_{\infty}$ as $t$ goes to infinity, which corresponds to $L$ in (7) and, with our hypothesis on $u_{0}$, the convergence (6) satisfies:

$$
\left|u(x, t)-w_{0}\left(|x|-c t+\frac{n-1}{c} \log t+L\right)\right| \leqslant c_{0} \frac{\log t}{t} .
$$

In a second section, we are interested in non-spherically symmetric perturbations of travelling waves in higher dimensions. Based on Uchiyama's work and a comparison theorem, a corollary on the Lyapunov stability of travelling waves against general small perturbations is first stated.

The only result so far concerning the long-time behaviour of non-spherically symmetric solutions is due to Jones [8]. He considered solutions $u(x, t)$ whose initial data $u_{0}$ have compact support, and he also assumed that $u(x, t)$ converges to 1 uniformly on compact sets as $t$ goes to infinity. He then showed that, if followed out in a radial direction at the correct speed $c$, the solution approaches the one-dimensional travelling wave, at least in shape. Moreover, for any $l \in(0,1)$ and any sufficiently large $t>0$, he proved that, for all point $P$ of the level surface $S_{l}(t)=\left\{x \in \mathbf{R}^{n} \mid u(x, t)=l\right\}$, the normal to $S_{l}(t)$ at $P$ must intersect the support of $u_{0}$. Obviously, this result implies that the surface $S_{l}(t)$ becomes rounder and rounder as $t$ goes to infinity. It is thus natural to expect spherically symmetric travelling waves to be asymptotically stable against any small non-symmetric perturbations. However, we prove in Section 2 that this is not the case. In the two-dimensional case, we give an example of nonspherically symmetric function $u_{0}$ close to a spherically symmetric wave such that the solution $u(x, t)$ of $(1)$ with initial data $u_{0}$ never approaches the family of spherically symmetric travelling waves. Indeed, the translate of the wave which is approached depends on the radial direction.

Subsequently, we require some more technical assumptions. For convenience, we choose to work in $\mathbf{R}^{2}$ so that polar coordinates are easier to handle. We assume that $F$ is in $\mathcal{C}^{3}(\mathbf{R})$ and satisfies the condition: $F^{(3)}(u) \leqslant 0$ for $u \in[0,1]$. In this case, we prove in Appendix $C$ that $\phi_{0}$ is log-concave, i.e., $\left(\phi_{0}^{\prime} / \phi_{0}\right)^{\prime}<0$. Finally, we also assume that every solution of the ODE, $u_{t}=F(u)$, is bounded uniformly in time. By the maximum principle, this easily means that for any bounded initial condition, the solution $u(x, t)$ is uniformly bounded in time. Example (2) for $F$ satisfies both conditions.

Precisely, we prove in the second section the following theorem:

Theorem 2. Assume $F$ is a "bistable" nonlinearity satisfying both above conditions. There exist positive constants $R_{0}^{\prime}, \delta_{0}^{\prime}, \eta, c_{0}$ such that if $u_{0} \in H^{1}\left(\mathbf{R}^{2}\right)$ satisfies

$$
\left\|u_{0}(x)-w_{0}(|x|-R)\right\|_{H^{1}\left(\mathbf{R}^{2}\right)} \leqslant \delta
$$

for some $\delta \leqslant \delta_{0}^{\prime}$ and some $R \geqslant R_{0}^{\prime}$ such that $R^{1 / 4} \delta \leqslant \eta$, then Eq. (1) has a unique solution $u \in C^{0}\left(\mathbf{R}^{+}, H^{1}\left(\mathbf{R}^{2}\right)\right)$ with initial data $u_{0}$. Moreover, there exist $\rho \in C^{0}\left(\mathbf{R}^{+}, H^{1}(0,2 \pi)\right)$ and $\rho_{\infty} \in L^{2}(0,2 \pi)$ such that

$$
\left\|u(r, \theta, t)-w_{0}(r-s(\theta, t))\right\|_{H^{1}\left(\mathbf{R}^{2}\right)} \leqslant \frac{c_{0}}{(R+c t)^{1 / 4}},
$$




$$
\begin{aligned}
& s(\theta, t)=R+c t-\frac{1}{c} \log \left(\frac{R+c t}{R}\right)+\rho(\theta, t), \\
& \lim _{t \rightarrow+\infty}\left\|\rho(\theta, t)-\rho_{\infty}(\theta)\right\|_{L^{2}(0,2 \pi)}=0,
\end{aligned}
$$

where $(r, \theta) \in \mathbf{R}^{+} \times(0,2 \pi)$ are the polar coordinates in $\mathbf{R}^{2}$.

This second theorem first illustrates Jones' theorem. Indeed, there exists a class of initial data for which solutions converge to a creased profile as $t$ goes to infinity. And, if followed out in a radial direction (i.e., for $\theta=$ constant), the solutions behave asymptotically as a one-dimensional travelling wave whose position $s(\theta, t)$ depends on the radial direction. Precisely, we show that $s(\theta, t)$ is given by (9), that $\rho(\theta, t)$ converges in the $L^{2}(0,2 \pi)$ norm to a function $\rho_{\infty}(\theta)$ and we give an example of initial data for which the solution does not converge to a spherically symmetric travelling wave, i.e., the corresponding function $\rho_{\infty}(\theta)$ is not constant. Moreover, we show that the set of all functions $\rho_{\infty}$ that can be constructed in that way, is dense in a ball of $H^{1}(0,2 \pi)$. Therefore, there exist a lot of asymptotic behaviours which look like a creased travelling front which never becomes round.

Finally, this theorem shows that the family of spherically symmetric travelling waves is not asymptotically stable for arbitrary perturbations: this means that the higher dimensional case $n \geqslant 2$ is very different from the one-dimensional case $n=1$ where the asymptotical stability of travelling waves has been widely proved.

Let us now make a few technical remarks on the statement of theorem 2 . We assume that the initial condition $u_{0}$ is close to a travelling wave ( $\delta \leqslant \delta_{0}^{\prime}$ small) whose interface $\left\{w_{0}=\frac{1}{2}\right\}$ is large enough $\left(R \geqslant R_{0}^{\prime}\right.$ large). The relation $R^{1 / 4} \delta \leqslant \eta$ should be a technical assumption and we do believe that it can be relaxed by changing the function spaces we use. Actually, we prove in this paper a stronger theorem (Theorem 2.5) where this constraint only appears on one part of the perturbation. We also show in this theorem that the perturbation decreases like $1 /(R+c t)^{1 / 4}$. This rate may not be optimal but shows the convergence of the solutions towards travelling fronts. Once more, we prove in Theorem 2.5 a more precise result where the dependance of the initial condition on the convergence rate is emphasized.

Notations. Throughout the paper, we use the following notations: $\|\cdot\|_{Z}$ is a norm in the Banach space $Z,|\cdot|$ is the usual norm in $\mathbf{R}$ and $x$ is a vector of $\mathbf{R}^{n}$ while $(r, \theta)$ are the polar coordinates in $\mathbf{R}^{2}$ where $r \geqslant 0, \theta \in[0,2 \pi)$. We also denote $c_{i}$ generic positive constants which may differ from place to place, even in the same chain of inequalities.

\section{Radial solutions}

The aim of this section is to prove Theorem 1, i.e., the stability of travelling waves against radial perturbations. Hence, we only work with spherically symmetric functions and we always use, for convenience, the notation $u(r, t)$ instead of $\tilde{u}(r, t)$ defined in the introduction.

For spherically symmetric solutions, Eq. (1) reduces to the following Cauchy problem:

$$
\begin{cases}u_{t}(r, t)=u_{r r}(r, t)+\frac{n-1}{r} u_{r}(r, t)+F(u(r, t)), & r>0, t>0, \\ u(r, 0)=u_{0}(r), & r>0, \\ \left.u_{r}\right|_{r=0}=0, & t \geqslant 0 .\end{cases}
$$

The Neumann boundary condition at zero is due to the regularity of the function $u(x, t), x \in \mathbf{R}^{n}$. In this section, we first write a decomposition of the solution $u(r, t)$ as Kapitula [10] did. Then, we study the new evolution equations in a moving frame to take advantage of spectral properties of the operator $L_{0}$ defined in the introduction. 


\subsection{A coordinate system}

We first need to define more precisely a spherically symmetric travelling wave in higher dimension. Since the function

$$
x \in \mathbf{R}^{n} \mapsto W(x, t)=w_{0}\left(|x|-R-c t+\frac{n-1}{c} \log \left(\frac{R+c t}{R}\right)\right)
$$

is not smooth at $x=0$, we have to modify $w_{0}$ in a function $w$ called also travelling wave or "modified wave".

Let $\chi \in C^{\infty}\left(\mathbf{R}^{+}\right)$so that $\chi(r) \equiv 0$ if $r \leqslant 1$ and $\chi(r) \equiv 1$ if $r \geqslant 2$, and define

$$
w(y, r)=1+\chi(r)\left(w_{0}(y)-1\right), \quad(y, r) \in \mathbf{R} \times \mathbf{R}^{+} .
$$

Then, $w(y, r)$ is identically equal to 1 if $r \leqslant 1$ and $w(y, r)=w_{0}(y)$ if $r \geqslant 2$. Note that $r$ is a positive parameter which flattens the wave around the origin. Then, for any $s \in \mathbf{R}, r \in \mathbf{R}^{+} \mapsto w(r-s, r)$ is a function of $Y=H^{1}\left(\mathbf{R}^{+}\right)$, equal to 1 near the origin and decreasing like the wave $w_{0}$ at infinity. In a similar way, $x \in \mathbf{R}^{n} \mapsto w(|x|-s,|x|)$ is a spherically symmetric function of $X$, equal to 1 near the origin and decreasing like the wave $w_{0}$ at infinity in all directions. We also define $\psi(y, r)=\bar{\alpha} \chi(r) \psi_{0}(y)$ where $\bar{\alpha}$ has been chosen in (5).

In a neighborhood of the wave $w$, it will be convenient to use a coordinate system given by $(v, s) \in Y \times \mathbf{R}$ with perturbations of the wave being given at any time by

$$
u(r)=w(r-s, r)+v(r), \quad r \geqslant 0,
$$

where $s$ is chosen so that $\int_{0}^{\infty} v(r) \psi(r-s, r) \mathrm{d} r=0$. We have decomposed the solution $u$ as a translate of the wave $w$ and a transversal perturbation $v$. The following lemma shows that this decomposition is always possible:

Lemma 1.1. There exist positive constants $R_{1}, \delta_{1}, K$ such that for any $R \geqslant R_{1}$ and any $\xi \in Y$ with $\|\xi\|_{Y} \leqslant \delta_{1}$, threre exists a unique pair $(v, \rho) \in Y \times \mathbf{R}$ such that

(i) $\|v\|_{Y}+|\rho| \leqslant K\|\xi\|_{Y}$,

(ii) $w(r-R, r)+\xi(r)=w(r-R-\rho, r)+v(r)$ for all $r \geqslant 0$,

(iii) $\int_{0}^{\infty} v(r) \psi(r-R-\rho, r) \mathrm{d} r=0$.

Proof. Define the operator $A: \mathbf{R} \times Y \rightarrow \mathbf{R}$ by

$$
A(\rho, \xi)=\int_{0}^{\infty} \xi(r) \psi(r-R-\rho, r) \mathrm{d} r+\rho \int_{0}^{\infty} \psi(r-R-\rho, r) \int_{0}^{1} w_{y}(r-R-\rho h, r) \mathrm{d} h \mathrm{~d} r .
$$

Since $A(0,0)=0$ and the derivative $A_{\rho}(0,0)=\bar{\alpha}^{2} \int_{-R}^{+\infty} \phi_{0} \psi_{0}(y) \chi^{2}(y+R) \mathrm{d} y \neq 0$ for $R \geqslant R_{1}$, by the implicit function theorem on Banach spaces, there exist a small neighborhood $\mathcal{V}=\mathcal{V}_{1} \times \mathcal{V}_{2}$ of $(0,0)$ in $\mathbf{R} \times Y$ a function $\rho(\xi): \mathcal{V}_{2} \mapsto \mathcal{V}_{1}$ such that $A(\rho(\xi), \xi)=0$ and $|\rho| \leqslant K\|\xi\|_{Y}$ for some $K>0$. This yields the spatial translational component $\rho$. Let $v(\cdot)=\xi(\cdot)+w(\cdot-R, \cdot)-w(\cdot-R-\rho(\xi), \cdot)$ a function of $Y$. Then, $\|v\|_{Y}+|\rho| \leqslant K\|\xi\|_{Y}$ for some $K>0$. As $A(\rho(\xi), \xi)=0$, and by Taylor's theorem, $\int_{0}^{\infty} v(r) \psi(r-R-\rho, r) \mathrm{d} r=0$. Then, $(v, \rho)$ satisfies the lemma if $\|\xi\|_{Y} \leqslant \delta_{1}$ where $\delta_{1}>0$ is sufficiently small so that $B_{Y}\left(0, \delta_{1}\right) \subset \mathcal{V}_{2}$.

Using the result of Lemma 1.1 , we can write for any $t \geqslant 0$ and some $R \geqslant R_{1}$,

$$
\begin{aligned}
& u(r, t)=w(r-s(t), r)+v(r, t), \quad r \geqslant 0, \\
& s(t)=R+c t-\frac{n-1}{c} \log \left(\frac{R+c t}{R}\right)+\rho(t),
\end{aligned}
$$




$$
\int_{0}^{\infty} v(r, t) \psi(r-s(t), r) \mathrm{d} r=0 .
$$

By Lemma 1.1, such a decomposition exists if, for all $t \geqslant 0$, the solution $u(r, t)$ is close to the wave, namely if $\|u(r, t)-w(r-s(t), r)\|_{Y} \leqslant \delta_{1}$. This assumption will be validated later by the proof of Theorem 1 . We are now going to work with these new variables $v$ and $\rho$ which are more convenient than $u$. We first give the equations they satisfy:

Substitute the decomposition (10) of the solution into Eq. (1) and use equation (3) satisfied by $w_{0}$ to get the evolution equation satisfied by $v$ :

$$
\begin{aligned}
& v_{t}=v_{r r}+\frac{n-1}{r} v_{r}+F^{\prime}\left(w_{0}(r-s(t))\right) v \\
& \quad+\left(\frac{n-1}{r}-\frac{n-1}{R+c t}+\rho^{\prime}(t)\right) w_{y}(r-s(t), r)+N+S, \quad r \geqslant 0, t>0, \\
& \begin{aligned}
& v(r, 0)=v_{0}(r), \quad r \geqslant 0, \\
&\left.v_{r}\right|_{r=0}=0, \quad t>0,
\end{aligned}
\end{aligned}
$$

where

$$
\begin{aligned}
& N=F(w+v)-F\left(w_{0}\right) \chi(r)-F^{\prime}\left(w_{0}\right) v \text { is the nonlinear term, } \\
& S=w_{r r}+2 w_{r y}+\frac{n-1}{r} w_{r} .
\end{aligned}
$$

The functions $w, w_{0}, \psi$ and their derivatives are taken at $(r-s(t), r)$ or $(r-s(t))$, depending if the wave is modified or not. Note the Neumann condition at zero $\left.v_{r}\right|_{r=0}=0$. Indeed, if $u(x)=\tilde{u}(|x|), u \in \mathcal{C}^{1}\left(\mathbf{R}^{2}\right)$ is equivalent to $\tilde{u} \in \mathcal{C}^{1}\left(\mathbf{R}^{+}\right)$and $\tilde{u}^{\prime}(0)=0$. As $u=w+v$ and $w$ is identically zero near the origin, the regularity of $u$ is forwarded to $v$ and $\left.v_{r}\right|_{r=0}=0$.

Derivating Eq. (11) with respect to $t$ and using Eqs. (8) and (12) satisfied by $s$ and $v$, we get the evolution equation satisfied by $\rho$ :

$$
\begin{aligned}
& \rho^{\prime}(t) \int_{0}^{\infty}\left(\psi w_{y}-v \psi_{y}\right) \mathrm{d} r=\int_{0}^{\infty}[v \Lambda-(N+S) \psi] \mathrm{d} r, \quad t>0, \\
& \rho(0)=\rho_{0},
\end{aligned}
$$

where

$$
\Lambda=\left(\frac{n-1}{R+c t}-\frac{n-1}{r}\right) \psi_{y}+\frac{n-1}{r^{2}} \psi+\left(\psi_{r r}+2 \psi_{y r}-\frac{n-1}{r} \psi_{r}\right)+\left(\psi_{y y}-c \psi_{y}+F^{\prime}\left(w_{0}\right) \psi .\right.
$$

The functions $\psi, w, w_{0}$ and their derivatives are taken at $(r-s(t), r)$ or $(r-s(t))$.

We first consider the initial value problem for Eqs. (12), (13):

Lemma 1.2. Fix $R>0$. There exist $\delta_{4}>0, T>0$ such that for any initial data $\left(v_{0}, \rho_{0}\right) \in Y \times \mathbf{R}$ with $\left\|v_{0}\right\|_{Y} \leqslant \delta \leqslant \delta_{4}$ and $\left|\rho_{0}\right| \leqslant \frac{1}{2}$, the integral equations corresponding to (12), (13) have a unique solution $(v, \rho) \in$ $C^{0}([0, T], Y \times \mathbf{R})$. In addition, $(v, \rho) \in C^{1}((0, T], Y \times \mathbf{R})$, and Eqs. (12), (13) are satisfied for $0<t \leqslant T$.

Proof. If $\left\|v_{0}\right\|_{Y} \leqslant \delta$ and $\delta \leqslant \delta_{4}$ is sufficiently small, then $\int_{0}^{\infty} \psi w_{y}-v \psi_{y} \mathrm{~d} r \neq 0$ and $\rho^{\prime}(t)$ can be expressed easily as a function of $v$ and $\rho$. Then, Eqs. (12), (13) can be written as follows: 


$$
\begin{aligned}
& \partial_{t}(v, \rho)=\bar{L}(v, \rho)+f(v, \rho, t), \\
& \left.v_{r}\right|_{r=0}=0, \\
& (v, \rho)(0)=\left(v_{0}, \rho_{0}\right),
\end{aligned}
$$

where

$$
\bar{L}(v, \rho)=(L v, 0)=\left(\partial_{r}^{2} v+\frac{n-1}{r} \partial_{r} v, 0\right) .
$$

As $\bar{L}$ generates a semigroup on $Y \times \mathbf{R}$ (see Lemma 1.5 for a detailed proof) and $f \in \mathcal{C}^{1}\left(Y \times \mathbf{R} \times \mathbf{R}^{+}\right.$), the integral equations corresponding to (12), (13) have a unique solution $(v, \rho) \in \mathcal{C}^{0}([0, T], Y \times \mathbf{R})$, see for instance [13]. In addition, this mild solution is classical and $(v, \rho) \in \mathcal{C}^{1}((0, T], Y \times \mathbf{R})$.

We now work on the two evolution equations (12), (13) to get information on the asymptotic behaviours of $v$ and $\rho$. Before stating our result, let us explain its content in a heuristic way. Consider first equation (12) for $v$. The leading term in the right-hand side is

$$
\left(\frac{n-1}{r}-\frac{n-1}{R+c t}+\rho^{\prime}(t)\right) w_{y}(r-s(t), r),
$$

which decays exponentially in time for any fixed $r>0$, but only like $(\log (R+c t)) /(R+c t)^{2}$ for $r=s(t)$. On the other hand, as we shall show in Section 1.2.3, the evolution operator generated by the time-dependent operator $\partial_{r}^{2}+\frac{n-1}{r} \partial_{r}+F^{\prime}\left(w_{0}(r-s(t))\right)$ is exponentially contracting in the space of functions $v$ satisfying (11). Therefore, we expect the solution $v$ of (12) to decay like $\log t / t^{2}$ as $t$ goes to infinity. As for $\rho$, we observe that Eq. (13) is close for large times to

$$
\rho^{\prime}(t)=\int_{0}^{\infty}\left[\left(\frac{n-1}{R+c t}-\frac{n-1}{r}\right) \psi_{y}+\frac{n-1}{r^{2}} \psi\right] v(r, t) \mathrm{d} r,
$$

since $\int_{0}^{\infty} \psi w_{y} \mathrm{~d} r$ is close to $\int_{\mathbf{R}} \psi_{0} \phi_{0} \mathrm{~d} x=1$. Thus, we also expect $\rho^{\prime}(t)$ to decrease at least like $\log t / t^{2}$ as $t$ goes to infinity. The following result shows that these heuristic considerations are indeed correct:

Theorem 1.3. There exist positive constants $R_{2}, \delta_{2}, c_{1}, c_{2}, \gamma_{0}$ such that, if $R \geqslant R_{2}$ and $\left(v_{0}, \rho_{0}\right) \in Y \times \mathbf{R}$ satisfy $\left\|v_{0}\right\|_{Y} \leqslant \delta_{2},\left|\rho_{0}\right| \leqslant \frac{1}{2}$, then Eqs. (12), (13) have a unique solution $(v, \rho) \in C^{0}([0,+\infty), Y \times \mathbf{R})$ with initial data $\left(v_{0}, \rho_{0}\right)$. In addition, $\rho \in C^{1}([0,+\infty), \mathbf{R})$ and

$$
\|v(t)\|_{Y}+\left|\rho^{\prime}(t)\right| \leqslant c_{1}\left\|v_{0}\right\|_{Y} \mathrm{e}^{-\gamma_{0} t}+c_{2} \frac{\log (R+c t)}{(R+c t)^{2}}, \quad t \geqslant 0 .
$$

Theorem 1.3 is a new version of Theorem 1 in the variables $v$ and $\rho$. We give right now the proof of Theorem 1 under the assumption that Theorem 1.3 is proved.

Proof of Theorem 1. Let $R_{2}, \delta_{2}, c_{1}, c_{2}, \gamma_{0}$ be as in Theorem 1.3 and $R_{1}, \delta_{1}, K$ be as in Lemma 1.1. Choose $R_{0}$ and $\delta_{0}$ so that:

$$
2 \delta_{0} \leqslant \delta_{1}, \quad 2 K \delta_{0} \leqslant \min \left(\delta_{2}, \frac{1}{2}\right), \quad R_{0} \geqslant \max \left(R_{2}, R_{1}\right), \quad c_{0} \mathrm{e}^{-\gamma_{1} R_{0}} \leqslant \delta_{0},
$$

where $c_{0}>0$ and $\gamma_{1}>0$ are chosen so that for any $R \geqslant 0$,

$$
\left\|w_{0}(r-R)-w(r-R, r)\right\|_{Y} \leqslant c_{0} \mathrm{e}^{-\gamma_{1} R} .
$$


Let $u_{0}: \mathbf{R}^{n} \rightarrow \mathbf{R}$ be a spherically symmetric function satisfying

$$
\left\|u_{0}(r)-w_{0}(r-R)\right\|_{Y} \leqslant \delta
$$

for some $R \geqslant R_{0}$ and $\delta \leqslant \delta_{0}$. Let $\xi(r)=u_{0}(r)-w(r-R, r), r \geqslant 0$. Then, $\xi \in Y$ and $\|\xi\|_{Y} \leqslant \delta+c_{0} \mathrm{e}^{-\gamma_{1} R} \leqslant$ $2 \delta_{0} \leqslant \delta_{1}$. Then, by Lemma 1.1 , there exists a unique pair $\left(v_{0}, \rho_{0}\right) \in Y \times \mathbf{R}$ such that:

(i) $\left\|v_{0}\right\|_{Y}+\left|\rho_{0}\right| \leqslant K\|\xi\|_{Y}$

(ii) $u_{0}(r)=w(r-R, r)+\xi(r)=w\left(r-R-\rho_{0}, r\right)+v_{0}(r)$ for all $r \geqslant 0$,

(iii) $\int_{0}^{\infty} v_{0}(r) \psi\left(r-R-\rho_{0}, r\right) \mathrm{d} r=0$.

As $R \geqslant R_{2}$ and $\left(v_{0}, \rho_{0}\right) \in Y \times \mathbf{R}$ satisfy $\left\|v_{0}\right\|_{Y} \leqslant \delta_{2}$ and $\left|\rho_{0}\right| \leqslant \frac{1}{2}$, it follows from Theorem 1.3 that Eqs. (12), (13) have a unique solution $(v, \rho) \in C^{0}([0,+\infty), Y \times \mathbf{R})$ with initial data $\left(v_{0}, \rho_{0}\right)$. In addition, $\rho \in C^{1}([0,+\infty), \mathbf{R})$ and

$$
\|v(t)\|_{Y}+\left|\rho^{\prime}(t)\right| \leqslant c_{1}\left\|v_{0}\right\|_{Y} \mathrm{e}^{-\gamma_{0} t}+c_{2} \frac{\log (R+c t)}{(R+c t)^{2}}, \quad t \geqslant 0 .
$$

Let $u(x, t)=w(|x|-s(t),|x|)+v(|x|, t), x \in \mathbf{R}^{n}$, where $s(t)$ is given by (8). Then, $u \in C^{0}([0,+\infty), X)$ is the unique solution of Eq. (1) with initial data $u_{0}$ and

$$
\begin{aligned}
& \left\|u(x, t)-w_{0}(|x|-s(t))\right\|_{X}+\left|\rho^{\prime}(t)\right| \\
& \quad \leqslant\|u(x, t)-w(|x|-s(t),|x|)\|_{X}+\left\|w(r-s(t), r)-w_{0}(r-s(t))\right\|_{Y}+\left|\rho^{\prime}(t)\right| \\
& \quad \leqslant c_{1}\left\|v_{0}\right\|_{Y} \mathrm{e}^{-\gamma_{0} t}+c_{2} \frac{\log (R+c t)}{(R+c t)^{2}}+c_{0} \mathrm{e}^{-\gamma_{1} s(t)} \\
& \quad \leqslant c_{1} K \delta \mathrm{e}^{-\gamma_{0} t}+c_{2} \frac{\log (R+c t)}{(R+c t)^{2}}+c_{1} K c_{0} \mathrm{e}^{-\gamma_{1} R-\gamma_{0} t}+c_{0} \mathrm{e}^{-\gamma_{1} s(t)} .
\end{aligned}
$$

Define $c_{1}^{\prime}=K c_{1}$ and $c_{2}^{\prime}$ so that for any $t \geqslant 0$, any $R \geqslant 0$,

$$
c_{2}+c_{1} c_{0} K \frac{\mathrm{e}^{-\gamma_{1} R-\gamma_{0} t}}{(\log (R+c t)) /(R+c t)^{2}}+c_{0} \frac{\mathrm{e}^{-\gamma_{1} s(t)}}{(\log (R+c t)) /(R+c t)^{2}} \leqslant c_{2}^{\prime} .
$$

Then,

$$
\left\|u(x, t)-w_{0}(|x|-s(t))\right\|_{X}+\left|\rho^{\prime}(t)\right| \leqslant c_{1}^{\prime} \delta \mathrm{e}^{-\gamma_{0} t}+c_{2}^{\prime} \frac{\log (R+c t)}{(R+c t)^{2}} .
$$

This ends the proof of Theorem 1.

\subsection{Estimates on the solutions $v$ and $\rho$}

Let us now prove Theorem 1.3. We begin with a proposition close to this theorem but local in time. We then show how Theorem 1.3 follows from this proposition.

Proposition 1.4. There exist positive constants $R_{3}, \delta_{3}, c_{1}, c_{2}, \gamma_{0}$ such that, if $R \geqslant R_{3}, T>0$ and $(v, \rho) \in$ $C^{0}([0, T], Y \times \mathbf{R})$ is any solution of $(12),(13)$ satisfying

$$
\|v(t)\|_{Y} \leqslant \delta_{3}, \quad|\rho(t)| \leqslant 1, \quad 0 \leqslant t \leqslant T
$$

then

$$
\|v(t)\|_{Y}+\left|\rho^{\prime}(t)\right| \leqslant c_{1}\left\|v_{0}\right\|_{Y} \mathrm{e}^{-\gamma_{0} t}+c_{2} \frac{\log (R+c t)}{(R+c t)^{2}}, \quad 0 \leqslant t \leqslant T .
$$


Proof of Theorem 1.3. Let $R_{3}, \delta_{3}, c_{1}, c_{2}, \gamma_{0}$ be as in Proposition 1.4 and choose positive constants $R_{2}, \delta_{2}$ so that $R_{2} \geqslant R_{3}$ and

$$
c_{1} \delta_{2}<\min \left(\frac{\delta_{3}}{2}, \frac{\gamma_{0}}{4}\right), \quad \delta_{2} \leqslant \min \left(\frac{\delta_{3}}{2}, \delta_{4}\right), \quad c_{2} \frac{\log R_{2}}{R_{2}^{2}}<\frac{\delta_{3}}{2}, \quad \frac{c_{2}}{c} \frac{1+\log R_{2}}{R_{2}}<\frac{1}{4} .
$$

Take $R \geqslant R_{2}$ and $\left(v_{0}, \rho_{0}\right) \in Y \times \mathbf{R}$ so that $\left\|v_{0}\right\|_{Y} \leqslant \delta_{2},\left|\rho_{0}\right| \leqslant \frac{1}{2}$. By Lemma 1.2 , let $(v, \rho) \in C^{0}\left(\left[0, T^{*}\right), Y \times \mathbf{R}\right)$ be the maximal solution of (12), (13) with initial data $\left(v_{0}, \rho_{0}\right)$. Define

$$
T=\sup \left\{\widetilde{T} \in\left[0, T^{*}\right) \mid\|v(t)\|_{Y} \leqslant \delta_{3} \text { and }|\rho(t)| \leqslant 1 \text { for any } t \in[0, \widetilde{T}]\right\} .
$$

Since $\delta_{2}<\delta_{3}$, it is clear that $T>0$. We claim that $T=T^{*}$, which also implies $T=T^{*}=+\infty$. Indeed, if $T<T^{*}$, it follows from Proposition 1.4 that for $t \in[0, T]$,

$$
\begin{aligned}
& \|v(t)\|_{Y} \leqslant c_{1}\left\|v_{0}\right\|_{Y} \mathrm{e}^{-\gamma_{0} t}+c_{2} \frac{\log (R+c t)}{(R+c t)^{2}} \leqslant c_{1} \delta_{2}+c_{2} \frac{\log R_{2}}{R_{2}^{2}}<\delta_{3}, \\
& |\rho(t)| \leqslant\left|\rho_{0}\right|+\int_{0}^{t}\left|\rho^{\prime}(s)\right| \mathrm{d} s \leqslant \frac{1}{2}+\frac{c_{1} \delta_{2}}{\gamma_{0}}+\frac{c_{2}}{c} \frac{1+\log R_{2}}{R_{2}}<1,
\end{aligned}
$$

which contradicts the definition of $T$. Thus $T=T^{*}=+\infty$. Since $\delta_{2}<\delta_{3}$, the inequality satisfied by $\|v(t)\|_{Y}+$ $\left|\rho^{\prime}(t)\right|$ is true for all $t \geqslant 0$ and Theorem 1.3 follows immediately from Proposition 1.4.

Let us now prove Proposition 1.4. We are first interested in the behaviour of $v$ which satisfies Eq. (12). The main idea is to work, as in one dimension, in the moving frame at speed $s(t)$ to get, in Eq. (12), a time independentoperator instead of $\partial_{r}^{2}+\frac{n-1}{r} \partial_{r}+F^{\prime}\left(w_{0}(r-s(t))\right)$. Therefore, we need to work on the whole real line which is invariant by translation. That is why we first extend $v$ to $\mathbf{R}$ by a function $z$ which is convenient, i.e., which decreases exponentially fast in time in the $H^{1}$ norm. Precisely, we already explained in a heuristic way that $v$ decreases exponentially fast as $t$ goes to infinity near $r=0$. Therefore, we first define a function $z$ equal to $v$ near the origin and then extend $v$ to $\mathbf{R}$ by $z$. We can then use theorems on spectral perturbations of operators, energy estimates and spectral decomposition to highlight the behaviour of $v$ in $X$. As Eqs. (12) and (13), satisfied by $v$ and $\rho$, are coupled, we need at the end to study the behaviour of $\rho$ as we explained before.

From now on, we fix $R>0$ (large), $0<\delta \leqslant \delta_{4}$ (small), and we assume that $(v, \rho) \in C^{0}([0, T], Y \times \mathbf{R})$ is a solution of (12), (13) satisfying

$$
\|v(t)\|_{Y} \leqslant \delta, \quad|\rho(t)| \leqslant 1, \quad 0 \leqslant t \leqslant T,
$$

for some $T>0$. We call these assumptions $(\mathrm{H})$.

\subsubsection{Localisation near $r=0$}

Let $\xi \in C^{\infty}\left(\mathbf{R}^{+}\right), R_{4} \geqslant 2$ and $\beta>0$ so that $\xi \equiv 1$ on $\left[0, R_{4}\right]$ and $\xi(r) \sim \mathrm{e}^{-\beta r}$ as $r$ goes to infinity. Let

$$
z(r, t)=\xi(r) v(r, t)
$$

for all $r \in \mathbf{R}^{+}$and $t \geqslant 0$. Then, $z$ is equal to $v$ near $r=0$ and satisfies

$$
\begin{aligned}
& z_{t}(r, t)=L_{1} z(r, t)+G_{1}(r, t), \quad r \geqslant 0, t>0, \\
& \left.z_{r}\right|_{r=0}=0, \quad t>0,
\end{aligned}
$$

where 


$$
\begin{aligned}
& L_{1}=\partial_{r}^{2}+\left(\frac{n-1}{r}+a(r)\right) \partial_{r}+b(r), \\
& G_{1}(r, t)=\left(F^{\prime}\left(w_{0}(r-s(t))\right)-h_{-}\right) \xi(r) v(r, t)+(S+N) \xi(r) \\
& +\left(\frac{n-1}{r}-\frac{n-1}{R+c t}+\rho^{\prime}(t)\right) w_{y}(r-s(t), r) \xi(r), \\
& a(r)=-2 \xi^{\prime}(r) / \xi(r), \\
& b(r)=2\left(\frac{\xi^{\prime}(r)}{\xi(r)}\right)^{2}-\frac{\xi^{\prime \prime}(r)}{\xi(r)}-\frac{n-1}{r} \frac{\xi^{\prime}(r)}{\xi(r)}+h_{-}, \\
& h_{-}=\inf \left[\lim _{y \rightarrow+\infty} F^{\prime}\left(w_{0}(y)\right), \lim _{y \rightarrow-\infty} F^{\prime}\left(w_{0}(y)\right)\right] \\
& =\inf \left(F^{\prime}(0), F^{\prime}(1)\right) \text {. }
\end{aligned}
$$

Note that $h_{-}<0$ and $b$ equals $h_{-}$near $r=0$. Therefore, by choice of appropriate $\beta, a(r)$ can be chosen small and $b(r) \leqslant-b_{0}<0$ for all $r \in \mathbf{R}^{+}$.

Lemma 1.5. Under assumptions $(\mathrm{H})$ for any $R \geqslant R_{4}, L_{1}$ generates an analytic semigroup on $Y$ and there exist positive constants $c_{0}, c_{1}, c_{2}, \gamma_{2}$ such that for any $t \in(0, T)$,

$$
\begin{aligned}
& \left\|\mathrm{e}^{t L_{1}}\right\|_{\mathcal{L}(Y)} \leqslant c_{0} \mathrm{e}^{-\gamma_{2} t} \\
& \left\|G_{1}(t)\right\|_{Y} \leqslant c_{1}(1+\delta) \mathrm{e}^{-\gamma_{2}(R+c t)}+c_{2} \delta\|v(t)\|_{Y} .
\end{aligned}
$$

Proof. We first study the behaviour of $\left\|G_{1}(t)\right\|_{Y}$ : it is a standard result that $w_{0}, \phi_{0}$ and $\psi_{0}$ decrease exponentially fast at infinity. Then, it comes that

$$
\begin{aligned}
& \left\|\left(F^{\prime}\left(w_{0}(r-s(t))\right)-h_{-}\right) \xi(r) v(r, t)\right\|_{Y} \leqslant c_{0} \delta \mathrm{e}^{-\gamma_{2}(R+c t)}, \\
& \|S\|_{Y} \leqslant c_{0} \mathrm{e}^{-\gamma_{2}(R+c t)}
\end{aligned}
$$

In addition, $N=\left[F(w+v)-F\left(w_{0}+v\right)\right]+\left[F\left(w_{0}+v\right)-F\left(w_{0}\right)-F^{\prime}\left(w_{0}\right) v\right]+F\left(w_{0}\right)(1-\chi(r))$ and

$$
\|N\|_{Y} \leqslant c_{0} \mathrm{e}^{-\gamma_{2}(R+c t)}+c_{0}\|v\|_{Y}^{2} \leqslant c_{0} \mathrm{e}^{-\gamma_{2}(R+c t)}+c_{0} \delta\|v\|_{Y} .
$$

Finally, we want to bound $\left\|\left((n-1) / r-(n-1) /(R+c t)+\rho^{\prime}(t)\right) w_{0}^{\prime}(r-s(t)) \chi(r) \xi(r)\right\|_{Y}$. As $R \geqslant R_{4}$, $s(t) \geqslant R_{4}$ and the particular case $r=s(t)$ explained in a heuristic way does not occur as $\xi(r)$ decays exponentially fast as $r$ goes to infinity. To conclude, we have to explain the bound of $\left|\rho^{\prime}(t)\right|$. Indeed, by Eq. (13),

$$
\left|\rho^{\prime}(t)\right| \leqslant c_{0}\left((1+\delta) \mathrm{e}^{-\gamma_{0}(R+c t)}+\delta \frac{\log (R+c t)}{(R+c t)^{2}}+\frac{\delta}{(R+c t)^{2}}+\delta\|v\|_{Y}\right)
$$

and

$$
\left\|\left(\frac{n-1}{r}-\frac{n-1}{R+c t}+\rho^{\prime}(t)\right) w_{0}^{\prime}(r-s(t)) \chi(r) \xi(r)\right\|_{Y} \leqslant c_{2}(1+\delta) \mathrm{e}^{-\gamma_{2}(R+c t)} .
$$

This ends the proof for $\left\|G_{1}\right\|_{Y}$.

On the other hand, the semi-group generated by $L_{1}$ on $Y$ is studied by energy estimates. Let $u$ be a solution of

$$
\begin{cases}u_{t}=L_{1} u, & r>0, t>0 \\ \left.u_{r}\right|_{r=0}=0, & t>0 \\ u(r, 0)=u_{0}(r), & r>0\end{cases}
$$

Let $I_{1}(t)=\frac{1}{2} \int_{0}^{\infty} u^{2} \mathrm{~d} r$ and $I_{2}(t)=\frac{1}{2} \int_{0}^{\infty} u_{r}^{2} \mathrm{~d} r$. Then, the derivatives with respect to $t$ of $I_{1}$ and $I_{2}$ satisfy 


$$
\begin{aligned}
& \dot{I}_{1}(t)=-2 I_{2}+(n-1) \int_{0}^{\infty} \frac{u u_{r}}{r} \mathrm{~d} r+\int_{0}^{\infty}\left(b-\frac{a^{\prime}}{2}\right) u^{2} \mathrm{~d} r, \\
& \dot{I}_{2}(t)=-\int_{0}^{\infty} u_{r r}^{2} \mathrm{~d} r-\frac{n-1}{2} \int_{0}^{\infty}\left(\frac{u_{r}}{r}\right)^{2} \mathrm{~d} r+\int_{0}^{\infty}\left(b+\frac{a^{\prime}}{2}\right) u_{r}^{2} \mathrm{~d} r-\int_{0}^{\infty} \frac{b^{\prime \prime}}{2} u^{2} \mathrm{~d} r .
\end{aligned}
$$

Let introduce $e>0, \varepsilon>0, I(t)=I_{1}(t)+e I_{2}(t)$, then

$$
\begin{aligned}
\dot{I}(t) \leqslant & \int_{0}^{\infty}\left(\left(b-\frac{a^{\prime}}{2}\right)-e \frac{b^{\prime \prime}}{2}+\frac{(n-1) \varepsilon}{2}\right) u^{2} \mathrm{~d} r \\
& +\int_{0}^{\infty}\left(-1+e\left(b+\frac{a^{\prime}}{2}\right)\right) u_{r}^{2} \mathrm{~d} r+\frac{n-1}{2}\left(\frac{1}{\varepsilon}-e\right) \int_{0}^{\infty}\left(\frac{u_{r}}{r}\right)^{2} \mathrm{~d} r .
\end{aligned}
$$

Choosing first $\varepsilon \ll 1$, then $e \gg 1$ depending on $\varepsilon$ and $\beta \ll 1$ depending on $e$, we obtain

$$
\begin{aligned}
& \left(b-\frac{a^{\prime}}{2}\right)-e \frac{b^{\prime \prime}}{2}+\frac{(n-1) \varepsilon}{2} \leqslant \frac{-\gamma_{2}}{2}<0, \\
& -1+e\left(b+\frac{a^{\prime}}{2}\right) \leqslant \frac{-\gamma_{2}}{2} e<0, \\
& \frac{1}{\varepsilon}-e \leqslant-1,
\end{aligned}
$$

where $\gamma_{2}=\left|b_{0}\right|$. It follows that $\dot{I}(t) \leqslant-\gamma_{2} I(t)$ and $\|u(t)\|_{Y} \leqslant c_{0} \mathrm{e}^{-\gamma_{2} t}\left\|u_{0}\right\|_{Y}$. This proves the lemma.

We shall use these calculations to get some further information on the behaviour of the semigroup generated by $L_{1}$ which are useful in the following sections. Let $\alpha(t)=\int_{0}^{\infty}\left(u_{r} / r\right)^{2} \mathrm{~d} r$. Then, according to (17),

$$
\frac{\mathrm{d}}{\mathrm{d} t}\left(\mathrm{e}^{\gamma_{2} t} I(t)\right)+\frac{n-1}{2} \mathrm{e}^{\gamma_{2} t} \alpha(t) \leqslant 0
$$

Integrating the latter inequality between $\sigma$ and $t$ and using Hölder's inequality, we obtain the following result for $\gamma$ defined in the introduction and any $(\sigma, t) \in(0, T)$ such that $\sigma \leqslant t$ :

$$
\int_{\sigma}^{t} \mathrm{e}^{-\gamma(t-s)}\left\|\frac{u_{r}}{r}(s)\right\|_{L^{2}\left(\mathbf{R}^{+}\right)} \mathrm{d} s \leqslant c_{3}\|u(\sigma)\|_{Y} \mathrm{e}^{-\left(\gamma_{2} / 2\right)(t-\sigma)} .
$$

In the same way, using convolution inequality $\|f * g\|_{L^{1}(\mathbf{R})} \leqslant\|f\|_{L^{1}(\mathbf{R})}\|g\|_{L^{1}(\mathbf{R})}$, we obtain for $\gamma^{\prime}<\gamma_{2}$,

$$
\int_{\sigma}^{t} \frac{\mathrm{e}^{-\gamma(t-s)}}{\sqrt{t-s}}\left\|\frac{u_{r}}{r}(s)\right\|_{L^{2}\left(\mathbf{R}^{+}\right)} \mathrm{d} s \leqslant c_{3}\|u(\sigma)\|_{Y} \mathrm{e}^{-\gamma^{\prime}(t-\sigma)} .
$$

The next lemma is a corollary of these calculations and will be used in the following to compute assymptotics of the solutions $(v, \rho)$.

Lemma 1.6. Under assumptions $(\mathrm{H})$ for any $R \geqslant R_{4}$, there exist positive constants $c_{0}, c_{1}, c_{2}, \gamma_{3}$ such that for any $t \in(0, T)$, 


$$
\begin{aligned}
& \int_{0}^{t} \mathrm{e}^{-\gamma(t-s)}\left\|\frac{z_{r}}{r}(s)\right\|_{L^{2}\left(\mathbf{R}^{+}\right)} \mathrm{d} s \leqslant c_{0}\left\|v_{0}\right\|_{Y} \mathrm{e}^{-\gamma_{3} t}+c_{1}(1+\delta) \mathrm{e}^{-\gamma_{3}(R+c t)}+c_{2} \delta \int_{0}^{t} \mathrm{e}^{-\frac{\gamma_{2}}{2}(t-s)}\|v(s)\|_{Y} \mathrm{~d} s, \\
& \int_{0}^{t} \frac{\mathrm{e}^{-\gamma(t-s)}}{\sqrt{t-s}}\left\|\frac{z_{r}}{r}(s)\right\|_{L^{2}\left(\mathbf{R}^{+}\right)} \mathrm{d} s \leqslant c_{0}\left\|v_{0}\right\|_{Y} \mathrm{e}^{-\gamma_{3} t}+c_{1}(1+\delta) \mathrm{e}^{-\gamma_{3}(R+c t)}+c_{2} \delta \int_{0}^{t} \mathrm{e}^{-\gamma^{\prime}(t-s)}\|v(s)\|_{Y} \mathrm{~d} s,
\end{aligned}
$$

where $z$ is defined in (15).

Proof. The proofs of these two inequalities are very similar. Therefore, we only prove the first one. We recall that $z(r, s)=\mathrm{e}^{s L_{1}} z_{0}+\int_{0}^{s} \mathrm{e}^{(s-\sigma) L_{1}} G_{1}(r, \sigma) \mathrm{d} \sigma$ for any $r \geqslant 0, s \geqslant 0$. Then,

$$
\begin{aligned}
& \int_{0}^{t} \mathrm{e}^{-\gamma(t-s)}\left\|\frac{z_{r}}{r}(s)\right\|_{L^{2}\left(\mathbf{R}^{+}\right)} \mathrm{d} s \\
& \quad \leqslant \int_{0}^{t} \mathrm{e}^{-\gamma(t-s)}\left\|\frac{\partial_{r}}{r} \mathrm{e}^{s L_{1}} z_{0}\right\|_{L^{2}\left(\mathbf{R}^{+}\right)} \mathrm{d} s+\int_{0}^{t} \mathrm{e}^{-\gamma(t-s)} \int_{0}^{s}\left\|\frac{\partial_{r}}{r} \mathrm{e}^{(s-\sigma) L_{1}} G_{1}(r, \sigma)\right\|_{L^{2}\left(\mathbf{R}^{+}\right)} \mathrm{d} \sigma \mathrm{d} s .
\end{aligned}
$$

The first term of the right-hand side is bounded by (18):

$$
\int_{0}^{t} \mathrm{e}^{-\gamma(t-s)}\left\|\frac{\partial_{r}}{r} \mathrm{e}^{s L_{1}} z_{0}\right\|_{L^{2}\left(\mathbf{R}^{+}\right)} \mathrm{d} s \leqslant c_{3} \mathrm{e}^{-\frac{\gamma_{2}}{2} t}\left\|z_{0}\right\|_{Y} .
$$

The second term is bounded by Fubini's theorem, (18) and Lemma 1.5:

$$
\begin{aligned}
\int_{0}^{t} \int_{0}^{s} \mathrm{e}^{-\gamma(t-s)}\left\|\frac{\partial_{r}}{r} \mathrm{e}^{(s-\sigma) L_{1}} G_{1}(r, \sigma)\right\|_{L^{2}\left(\mathbf{R}^{+}\right)} \mathrm{d} \sigma \mathrm{d} s & \leqslant \int_{0}^{t} c_{3} \mathrm{e}^{-\left(\gamma_{2} / 2\right)(t-\sigma)}\left\|G_{1}(r, \sigma)\right\|_{Y} \mathrm{~d} \sigma \\
& \leqslant c_{1}(1+\delta) \mathrm{e}^{-\gamma_{3}(R+c t)}+c_{2} \delta \int_{0}^{t} \mathrm{e}^{-\left(\gamma_{2} / 2\right)(t-\sigma)}\|v(\sigma)\|_{Y} \mathrm{~d} \sigma .
\end{aligned}
$$

This ends the proof of Lemma 1.6.

Corollary 1.7. Under assumptions $(\mathrm{H})$ for any $R \geqslant R_{4}$, the behaviour of $z$ is a result of Lemma 1.5 . Indeed, there exist positive constants $c_{1}, c_{2}, c_{3}$ such that for any $t \in(0, T)$,

$$
\|z(t)\|_{Y} \leqslant c_{1}\left\|v_{0}\right\|_{Y} \mathrm{e}^{-\gamma_{2} t}+c_{2}(1+\delta) \mathrm{e}^{-\gamma_{2}(R+c t)}+c_{3} \delta \int_{0}^{t} \mathrm{e}^{-\gamma_{2}(t-s)}\|v(s)\|_{Y} \mathrm{~d} s .
$$

\subsubsection{Extension to the real line}

As we said before, we need to work on the whole real line and therefore to extend $v$ for $r<0$. Let

$$
\tilde{z}(r, t)= \begin{cases}z(-r, t) & \text { if } r<0 \\ v(r, t) & \text { if } r \geqslant 0\end{cases}
$$

Then, $\tilde{z}$ is smooth in $\mathbf{R}$ and satisfies for any $r \in \mathbf{R}$, 


$$
\begin{aligned}
\tilde{z}_{t}(r, t)= & \tilde{z}_{r r}(r, t)+\frac{n-1}{r} \tilde{z}_{r}(r, t)+F^{\prime}\left(w_{0}(r-s(t))\right) \tilde{z}(r, t) \\
& +\left(\frac{n-1}{r}-\frac{n-1}{R+c t}+\rho^{\prime}(t)\right) w_{0}^{\prime}(r-s(t)) \chi(r)+\tilde{N}+G_{2}(r, t),
\end{aligned}
$$

where

$$
\begin{aligned}
& \tilde{N}= \begin{cases}N & \text { if } r \geqslant 0 \\
N \xi(|r|) & \text { if } r<0\end{cases} \\
& G_{2}(r, t)= \begin{cases}S \quad & \text { if } r \geqslant 0 \\
a z_{r}+\left(b-h_{-}\right) z+S \xi(|r|)+\left(F^{\prime}\left(w_{0}(|r|-s(t))\right)-F^{\prime}\left(w_{0}(r-s(t))\right)\right) z(|r|, t) & \\
& +\left(\frac{n-1}{|r|}-\frac{n-1}{R+c t}+\rho^{\prime}(t)\right) w_{0}^{\prime}(|r|-s(t)) \chi(|r|) \xi(|r|) \\
- & \left(\frac{n-1}{r}-\frac{n-1}{R+c t}+\rho^{\prime}(t)\right) w_{0}^{\prime}(r-s(t)) \chi(r) \xi(r) \quad \text { if } r \leqslant 0 .\end{cases}
\end{aligned}
$$

Using Lemma 1.5 and Corollary 1.7, we have the following lemma:

Lemma 1.8. Under assumptions $(\mathrm{H})$ with $R \geqslant R_{4}$, there exist positive constants $c_{1}, c_{2}, c_{3}$ such that for any $t \in(0, T)$,

$$
\left\|G_{2}(t)\right\|_{L^{2}(\mathbf{R})} \leqslant c_{1}\left\|v_{0}\right\|_{Y} \mathrm{e}^{-\gamma_{2} t}+c_{2}(1+\delta) \mathrm{e}^{-\gamma_{2}(R+c t)}+c_{3} \delta \int_{0}^{t} \mathrm{e}^{-\gamma_{2}(t-s)}\|v(s)\|_{Y} \mathrm{~d} s .
$$

\subsubsection{Moving frame}

In order to take advantage of spectral properties of the time independent operator $L_{0}$, it is convenient to work in the moving frame with speed $s(t)$. So let $\bar{z}(r-s(t), t)=\tilde{z}(r, t)$ and $G_{3}(r-s(t), t)=G_{2}(r, t)$. Then, $\bar{z}$ satisfies an equation similar to (19). As $\eta(t)=\int_{\mathbf{R}} \bar{z}(y, t) \psi_{0}(y) \mathrm{d} y=\int_{\mathbf{R}} \tilde{z}(r, t) \psi_{0}(r-s(t)) \mathrm{d} r$ is nonzero in general, $\bar{z}$ does not belong to $\mathcal{R}$. We recall that $\mathcal{R}$ has been defined in the introduction as the supplementary of the spectral subspace corresponding to the eigenvalue 0 of the operator $L_{0}$ in $L^{2}(\mathbf{R})$. As $L_{0}=\partial_{y}^{2}+c \partial_{y}+F^{\prime}\left(w_{0}\right)$ has interesting spectral properties in $\mathcal{R}$, it is convenient to use the following spectral decomposition:

$$
\bar{z}(y, t)=\eta(t) \phi_{0}(y)+r(y, t), \quad \text { where } r \in \mathcal{R} .
$$

Note that this $r \in \mathcal{R}$ is different from the $r \in \mathbf{R}^{+}$used so far. Before going on, notice that $\eta(t)$ decreases exponentially fast in time: $|\eta(t)| \leqslant c_{0} \mathrm{e}^{-\gamma_{4}(R+c t)}$ for $\gamma_{4}>0$, and let introduce a few notations. Let $\zeta \in C_{0}^{\infty}(\mathbf{R})$, positive, even, which satisfies $\zeta \equiv 1$ on $\left[-R_{4}, R_{4}\right]$ and $\zeta \equiv 0$ on $\left[-R_{4}-1, R_{4}+1\right]^{c}$.

We decompose the nonlinear terms as follows: $\widetilde{N}=N_{1}+N_{2}$ where

$$
N_{1}=F(w+r)-F\left(w_{0}\right) \chi(y+s(t))-F^{\prime}\left(w_{0}\right) r \quad \text { and } \quad N_{2}=\tilde{N}-N_{1} .
$$

Then,

$$
\begin{aligned}
& \left\|N_{1}\right\|_{L^{2}} \leqslant c_{0}\|r\|_{Y}^{2}+c_{0} \mathrm{e}^{-\gamma_{2}(R+c t)}, \\
& \left\|N_{2}\right\|_{L^{2}} \leqslant c_{0}|\eta(t)| .
\end{aligned}
$$

Substitute the decomposition (20) into Eq. (19) to get:

$$
\begin{aligned}
& r_{t}(y, t)=L_{2} r(y, t)+Q\left(G_{4}\right)(y, t), \quad t \geqslant 0, y \in \mathbf{R}, \\
& \int_{\mathbf{R}} r(y, t) \psi_{0}(y) \mathrm{d} y=0, \quad t>0,
\end{aligned}
$$


where

$$
\begin{aligned}
L_{2} & =\partial_{y}^{2}+c \partial_{y}+F^{\prime}\left(w_{0}\right)+Q\left(N_{1}+(1-\zeta) G_{5}\right) \\
G_{5} & =\left(\frac{n-1}{y+s(t)}-\frac{n-1}{R+c t}+\rho^{\prime}(t)\right) r_{y}(y, t) \\
G_{4} & =G_{3}(y, t)+N_{2}+\zeta G_{5}(y, t)+\left(\frac{n-1}{y+s(t)}-\frac{n-1}{R+c t}+\rho^{\prime}(t)\right)\left(\eta(t) \phi_{0}^{\prime}(y)+\xi(y+s(t)) \phi_{0}(y)\right) .
\end{aligned}
$$

We recall that $Q$ is a projector onto $\mathcal{R}$ defined in the introduction.

Lemma 1.9. There exist positive constants $R_{5}, \delta_{5}$ such that under assumptions $(\mathrm{H})$ with $R \geqslant R_{5}$ and $\delta \leqslant \delta_{5}, L_{2}$ generates a family of evolution operators $A(t, s)$ on $\mathcal{R}$ which satisfies

$$
\|A(t, s)\|_{\mathcal{L}(\mathcal{R})} \leqslant c_{0} \mathrm{e}^{-\gamma(t-s)}, \quad 0 \leqslant s \leqslant t
$$

Proof. Let $L_{0}=\partial_{y}^{2}+c \partial_{y}+F^{\prime}\left(w_{0}\right)$ defined on $\mathcal{R}$. Then $\left.\left.\sigma\left(L_{0 \mid \mathcal{R}}\right) \subset\right]-\infty ;-\gamma\right], \gamma>0$ and $L_{0}$ generates an analytic semi-group on $\mathcal{R}$ which satisfies $\left\|\mathrm{e}^{t L_{0}}\right\|_{\mathcal{L}(\mathcal{R})} \leqslant c_{0} \mathrm{e}^{-\gamma t}$ and $\mathcal{R}^{1 / 2} \equiv D\left(L_{0}^{1 / 2}\right)=H^{1}(\mathbf{R})$, see for instance [13]. Let

$$
\begin{aligned}
B: \mathbf{R}^{+} & \longrightarrow \mathcal{L}\left(H^{1}(\mathbf{R}), L^{2}(\mathbf{R})\right) \\
t & \longmapsto B(t): H^{1}(\mathbf{R}) \\
r & \mapsto L^{2}(\mathbf{R}) \\
r & \mapsto\left(N_{1}+(1-\zeta) G_{5}\right)
\end{aligned}
$$

We want to prove that $B$ is a small perturbation of the operator $L_{0}$ which does not affect its exponential decrease. As $\|B(t)\|_{\mathcal{L}\left(H^{1}, L^{2}\right)} \leqslant c_{0}((n-1) / R+\delta)$, Appendix A ends the proof, namely there exist some $R_{5} \gg 1$ and some $\delta_{5}>0$ so that for all $R \geqslant R_{5}$ and $\delta \leqslant \delta_{5}, L_{2}$ generates a family of evolution operators $A(t, s)$ on $\mathcal{R}$ which satisfies Lemma 1.9 for a slightly different $\gamma$.

Lemma 1.10. Under hypothesis $(\mathrm{H})$ with $R \geqslant R_{4}$, there exist positive constants $c_{i}, i=0, \ldots, 5$, and $\gamma_{5}$ such that for any $t \in(0, T)$,

$$
\begin{aligned}
\left\|Q\left(G_{4}\right)(t)\right\|_{L^{2}(\mathbf{R})} \leqslant & c_{0}\left\|v_{0}\right\|_{Y} \mathrm{e}^{-\gamma_{5} t}+c_{1}(1+\delta) \mathrm{e}^{-\gamma_{5}(R+c t)}+c_{3} \frac{\log (R+c t)}{(R+c t)^{2}} \\
& +c_{2} \delta \int_{0}^{t} \mathrm{e}^{-\gamma_{2}(t-s)}\|v(s)\|_{Y} \mathrm{~d} s+c_{4}\left|\rho^{\prime}(t)\right|+c_{5}\left\|\frac{z_{r}}{r}(t)\right\|_{L^{2}\left(\mathbf{R}^{+}\right)}
\end{aligned}
$$

Proof. As $G_{4}$ is given by (22), the first two terms have already been studied in Lemma 1.8 and (21):

$$
\begin{aligned}
& \left\|Q\left(G_{3}\right)(t)\right\|_{L^{2}(\mathbf{R})} \leqslant\left\|G_{2}(t)\right\|_{L^{2}\left(\mathbf{R}^{+}\right)} \\
& \qquad c_{1}\left\|v_{0}\right\|_{Y} \mathrm{e}^{-\gamma_{2} t}+c_{2}(1+\delta) \mathrm{e}^{-\gamma_{2}(R+c t)}+c_{3} \delta \int_{0}^{t} \mathrm{e}^{-\gamma_{2}(t-s)}\|v(s)\|_{Y} \mathrm{~d} s, \\
& \left\|Q\left(N_{2}\right)(t)\right\|_{L^{2}} \leqslant c_{0}|\eta(t)| \leqslant c_{0} \mathrm{e}^{-\gamma_{4}(R+c t)} .
\end{aligned}
$$

The last terms will be cut into four parts with the cut-off $\zeta$. As

$$
\left(\frac{n-1}{y+s(t)}-\frac{n-1}{R+c t}+\rho^{\prime}(t)\right) \xi(y+s(t)) \phi_{0}(y) \zeta(y+s(t))=0
$$


by definition of $\xi$ and $\zeta$, we obtain

$$
\left\|\left(\frac{n-1}{y+s(t)}-\frac{n-1}{R+c t}+\rho^{\prime}(t)\right) \xi(y+s(t)) \phi_{0}(y)\right\|_{L^{2}} \leqslant c_{0} \frac{\log (R+c t)}{(R+c t)^{2}}+c_{1}\left|\rho^{\prime}(t)\right| .
$$

In the same way, we get

$$
\left\|\left(\frac{n-1}{y+s(t)}-\frac{n-1}{R+c t}+\rho^{\prime}(t)\right) \eta(t) \phi_{0}^{\prime}(y)(1-\zeta(y+s(t)))\right\|_{L^{2}} \leqslant c_{2}(1+\delta) \mathrm{e}^{-\gamma_{4}(R+c t)} .
$$

Finally, we join the last two terms:

$$
\begin{aligned}
& \left\|\zeta G_{5}+\left(\frac{n-1}{y+s(t)}-\frac{n-1}{R+c t}+\rho^{\prime}(t)\right) \eta(t) \phi_{0}^{\prime}(y) \zeta(y+s(t))\right\|_{L^{2}(\mathbf{R})} \\
& \leqslant\left\|\left(\frac{n-1}{r}-\frac{n-1}{R+c t}+\rho^{\prime}(t)\right) \zeta(r) \tilde{z}_{r}(r, t)\right\|_{L^{2}(\mathbf{R})} \\
& \leqslant c_{2}\left\|\frac{z_{r}(r, t)}{r}\right\|_{L^{2}\left(\mathbf{R}^{+}\right)}+\left(\frac{n-1}{R}+c_{0} \delta+c_{1}\right)\|z(r, t)\|_{Y}
\end{aligned}
$$

as $\tilde{z}=z=v$ on $\left[0, R_{4}\right]$. By Corollary 1.7 , we conclude that:

$$
\begin{aligned}
& \left\|\zeta G_{5}+\left(\frac{n-1}{y+s(t)}-\frac{n-1}{R+c t}+\rho^{\prime}(t)\right) \eta(t) \phi_{0}^{\prime}(y) \zeta(y+s(t))\right\|_{L^{2}(\mathbf{R})} \\
& \quad \leqslant c_{0}\left\|v_{0}\right\|_{Y} \mathrm{e}^{-\gamma_{2} t}+c_{1}(1+\delta) \mathrm{e}^{-\gamma_{2}(R+c t)}+c_{2}\left\|\frac{z_{r}(r, t)}{r}\right\|_{L^{2}\left(\mathbf{R}^{+}\right)}+c_{3} \delta \int_{0}^{t} \mathrm{e}^{-\gamma_{2}(t-s)}\|v(s)\|_{Y} \mathrm{~d} s .
\end{aligned}
$$

Define $\gamma_{5}=\inf \left\{\gamma_{2}, \gamma_{4}\right\}$. This ends the proof.

Corollary 1.11. Under assumptions $(\mathrm{H})$ with $R \geqslant \max \left(R_{4}, R_{5}\right)$ and $\delta \leqslant \delta$, there exist positive constants $c_{i}$, $i=1, \ldots, 4$ and $\gamma_{7}, \gamma^{\prime}$ such that for any $t \in(0, T)$,

$$
\|r(t)\|_{H^{1}(\mathbf{R})} \leqslant c_{1}\left\|r_{0}\right\|_{H^{1}(\mathbf{R})} \mathrm{e}^{-\gamma_{7} t}+c_{2}(1+\delta) \mathrm{e}^{-\gamma_{7}(R+c t)}+c_{3} \frac{\log (R+c t)}{(R+c t)^{2}}+c_{4} \int_{0}^{t} \frac{\mathrm{e}^{-\gamma^{\prime}(t-s)}}{\sqrt{(t-s)}}\left|\rho^{\prime}(s)\right| \mathrm{d} s .
$$

Proof. We first want to bound the $L^{2}$ norm of $r$. As a consequence of Lemmas 1.9, 1.10 and 1.6, we get for any $t \in(0, T)$,

$$
\begin{aligned}
\|r(t)\|_{L^{2}(\mathbf{R})} \leqslant & c_{1}\left\|r_{0}\right\|_{H^{1}(\mathbf{R})} \mathrm{e}^{-\gamma_{6} t}+c_{2}(1+\delta) \mathrm{e}^{-\gamma_{6}(R+c t)}+c_{3} \frac{\log (R+c t)}{(R+c t)^{2}} \\
& +c_{4} \int_{0}^{t} \mathrm{e}^{-\gamma(t-s)}\left|\rho^{\prime}(s)\right| \mathrm{d} s+c_{5} \delta \int_{0}^{t} \mathrm{e}^{-\gamma_{6}(t-s)}\|r(s)\|_{H^{1}(\mathbf{R})} \mathrm{d} s .
\end{aligned}
$$

In order to bound the $H^{1}$ norm of $r$, we recall that $r_{t}=L_{2} r+Q\left(G_{4}\right)$ and $L_{2}=L_{0}+B(t)$. According to Lemma 1.9, operator $B(t)$ is a small perturbation of $L_{0}$. Then, the Banach space $\mathcal{R}^{1 / 2}$ can be defined by $D\left(A^{1 / 2}\right)$ as well as $D\left(L_{0}^{1 / 2}\right)$, and the graph norms are equivalent. Thus, $\left\|\partial_{x} A(t, s)\right\|_{\mathcal{L}(\mathcal{R})} \leqslant c_{0} \mathrm{e}^{-\gamma(t-s) / \sqrt{t-s}}$. In addition, 
$r(y, t)=A(t, 0) r_{0}(y)+\int_{0}^{t} A(t, s) Q\left(G_{4}\right)(y, s) \mathrm{d} s$. Derivating this last expression with respect to $y$ and bounding the $L^{2}$ norm, we get:

$$
\left\|\partial_{y} r(t)\right\|_{L^{2}(\mathbf{R})} \leqslant c_{0}\left\|r_{0}\right\|_{H^{1}(\mathbf{R})} \mathrm{e}^{-\gamma t}+\int_{0}^{t} \frac{\mathrm{e}^{-\gamma(t-s)}}{\sqrt{t-s}}\left\|Q\left(G_{4}\right)(s)\right\|_{L^{2}} \mathrm{~d} s .
$$

Finally, by (23) and Lemmas 1.10 and 1.6, we get

$$
\begin{aligned}
\|r(t)\|_{H^{1}(\mathbf{R})} \leqslant & c_{1}\left\|r_{0}\right\|_{H^{1}(\mathbf{R})} \mathrm{e}^{-\gamma_{6} t}+c_{2}(1+\delta) \mathrm{e}^{-\gamma_{6}(R+c t)}+c_{3} \frac{\log (R+c t)}{(R+c t)^{2}} \\
& +c_{4} \int_{0}^{t} \frac{\mathrm{e}^{-\gamma(t-s)}}{\sqrt{t-s}}\left|\rho^{\prime}(s)\right| \mathrm{d} s+c_{5} \int_{0}^{t} \frac{\mathrm{e}^{-\gamma(t-s)}}{\sqrt{t-s}}\|r(s)\|_{H^{1}} \mathrm{~d} s .
\end{aligned}
$$

Indeed, by Fubini's theorem and one integration by parts,

$$
\int_{0}^{t} \frac{\mathrm{e}^{-\gamma(t-s)}}{\sqrt{t-s}} \int_{0}^{s} \mathrm{e}^{-\gamma_{2}(s-\sigma)}\|v(\sigma)\|_{Y} \mathrm{~d} \sigma \mathrm{d} s \leqslant c_{0} \int_{0}^{t} \mathrm{e}^{-\gamma^{\prime}(t-s)}\|v(s)\|_{Y} \mathrm{~d} s .
$$

Gronwall's lemma ends the proof.

Corollary 1.12. Under the same assumptions $(\mathrm{H})$ with $R \geqslant \max \left(R_{4}, R_{5}\right)$ and $\delta \leqslant \delta_{5}$, there exist positive constants $c_{i}, i=1, \ldots, 3$, such that for any $t \in(0, T)$,

$$
\|v(t)\|_{Y} \leqslant c_{1}\left\|v_{0}\right\|_{Y} \mathrm{e}^{-\gamma_{8} t}+c_{2}(1+\delta) \mathrm{e}^{-\gamma_{8}(R+c t)}+c_{3} \frac{\log (R+c t)}{(R+c t)^{2}}+\int_{0}^{t} \frac{\mathrm{e}^{-\gamma(t-s)}}{\sqrt{(t-s)}}\left|\rho^{\prime}(s)\right| \mathrm{d} s .
$$

\subsubsection{Conclusion}

Proof of Proposition 1.4. Take $R_{3}=\max \left\{R_{4}, R_{5}\right\}$ and $\delta_{3}=\inf \left\{\delta_{4}, \delta_{5}\right\}$. Let $T>0, \delta \leqslant \delta_{3}$ and $R \geqslant R_{3}$. Consider $(v, \rho) \in \mathcal{C}^{0}([0, T], Y \times \mathbf{R})$ any solution of $(12,13)$ satisfying

$$
\|v\|_{Y} \leqslant \delta, \quad|\rho(t)| \leqslant 1, \quad 0 \leqslant t \leqslant T .
$$

Then, assumptions $(\mathrm{H})$ are valid and by inequality (16), Corollary 1.12 and Gronwall's lemma, there exist positive constants $c_{1}, c_{2}, \gamma_{0}$ such that

$$
\|v(t)\|_{Y}+\left|\rho^{\prime}(t)\right| \leqslant c_{1}\left\|v_{0}\right\|_{Y} \mathrm{e}^{-\gamma_{0} t}+c_{2} \frac{\log (R+c t)}{(R+c t)^{2}}, \quad 0 \leqslant t \leqslant T .
$$

This ends the proof of Proposition 1.4.

\section{Nonradial solutions}

In this section, we deal with nonradial solutions of Eq. (1). We prove, in this case, that travelling waves are Lyapunov stable but not necessarily asymptotically stable for general (i.e., nonnecessarily spherically symmetric) perturbations. In the first part of this section, we explain how the Lyapunov stability follows from Uchiyama's proposition and the maximum principle. In the second part, we prove Theorem 2. To this end, we introduce some energy functionals which enable us to rule out the asymptotic stability of travelling waves against arbitrary small perturbations. In particular, we give an example in $\mathbf{R}^{2}$ of an initial data $u_{0}$ close to a travelling wave which converges to a nonradial profile as $t$ goes to infinity. 


\subsection{Lyapunov stability}

In the first section, we proved in Theorem 1.3 the local stability of travelling waves in $X$, i.e., among radial perturbations. Note that Uchiyama [16] proved a similar result in the $L^{\infty}$ norm in her Lemma 4.5 without any information on the decay rate of the perturbation. Using comparison theorem, we show easily the Lyapunov stability of travelling waves against arbitrary small perturbations.

Proposition 2.1. For any $\varepsilon>0$, there exist positive constants $R_{0}, \delta$ such that if $u_{0}: \mathbf{R}^{n} \rightarrow \mathbf{R}$ is a spherically symmetric function satisfying

$$
\left\|u_{0}(x)-w_{0}(|x|-R)\right\|_{L^{\infty}\left(\mathbf{R}^{n}\right)} \leqslant \delta
$$

for some $R \geqslant R_{0}$, then Eq. (1) has a unique solution $u \in C^{0}\left(\mathbf{R}^{+}, L^{\infty}\left(\mathbf{R}^{n}\right)\right)$ with initial data $u_{0}$ and for all $t \in \mathbf{R}^{+}$,

$$
\left\|u(x, t)-w_{0}(|x|-\bar{s}(t))\right\|_{L^{\infty}\left(\mathbf{R}^{n}\right)} \leqslant \varepsilon
$$

where $\bar{s}(t)=R+c t-\frac{n-1}{c} \log (c(R+c t) / R)$.

Proof. See Uchiyama [16], Lemma 4.5.

Corollary 2.2. For any $\varepsilon>0$, there exist positive constants $R_{0}, \delta$ such that if $u_{0}: \mathbf{R}^{n} \rightarrow \mathbf{R}$ satisfies

$$
\left\|u_{0}(x)-w_{0}(|x|-R)\right\|_{L^{\infty}\left(\mathbf{R}^{n}\right)} \leqslant \delta
$$

for some $R \geqslant R_{0}$, then Eq. (1) has a unique solution $u \in \mathcal{C}^{0}\left(\mathbf{R}^{+}, L^{\infty}\left(\mathbf{R}^{n}\right)\right)$ with initial data $u_{0}$ and for all $t \in \mathbf{R}^{+}$,

$$
\left\|u(x, t)-w_{0}(|x|-\bar{s}(t))\right\|_{L^{\infty}\left(\mathbf{R}^{n}\right)} \leqslant \varepsilon
$$

where $\bar{s}(t)=R+c t-\frac{n-1}{c} \log \left(\frac{R+c t}{R}\right)$.

Proof. Let $u(x, t), u_{1}(x, t), u_{2}(x, t)$ be the solutions of Eq. (1) with initial data $u_{0}, w_{0}(|x|-R)-\delta, w_{0}(|x|-R)+$ $\delta$ respectively. Then, combining the maximum principle and Proposition 2.1, we have $u_{1}(x, t) \leqslant u(x, t) \leqslant u_{2}(x, t)$ on $\mathbf{R}^{n} \times \mathbf{R}^{+}$and $\left\|u(x, t)-w_{0}(|x|-\bar{s}(t))\right\|_{L^{\infty}\left(\mathbf{R}^{n}\right)} \leqslant \varepsilon$. This ends the proof.

\subsection{Energy estimates}

In order to prove Theorem 2 about nonradial profiles, we need to control the perturbation of the wave and in particular the shape of the interface. We proceed as in the first section: we decompose the solution $u(x, t)$ as a translate of the wave and a transversal perturbation. We use the same notations as in Section 1. As is explained in the introduction, we restrict ourselves for convenience in the two-dimensional case, and we use polar coordinates $(r, \theta) \in \mathbf{R}^{+} \times[0,2 \pi)$ in $\mathbf{R}^{2}$. Define the open set $\Omega=\mathbf{R}^{+*} \times(0,2 \pi)$ and the measure $\mathrm{d} \nu=r \mathrm{~d} r \mathrm{~d} \theta$. We need to introduce some Banach spaces adapted to these new variables:

$$
\begin{aligned}
& W=\left\{v(r, \theta) \in H_{\mathrm{loc}}^{1}(\Omega) \mid v, v_{r}, \frac{v_{\theta}}{r} \in L^{2}(\Omega, \mathrm{d} v) \text { and } v(r, 0)=v(r, 2 \pi) \text { in } L_{\mathrm{loc}}^{2}\left(\mathbf{R}^{+}, \mathrm{d} r\right)\right\}, \\
& Z=\left\{\rho(\theta) \in H^{1}(0,2 \pi) \mid \rho(0)=\rho(2 \pi)\right\} .
\end{aligned}
$$

We also define the associated norms:

$$
\|v\|_{W}=\left(\int_{\Omega}\left(v^{2}+v_{r}^{2}+\frac{v_{\theta}^{2}}{r^{2}}\right) \mathrm{d} v\right)^{1 / 2}
$$




$$
\|\rho\|_{Z}=\left(\int_{0}^{2 \pi}\left(\rho^{2}+\rho_{\theta}^{2}\right) \mathrm{d} \theta\right)^{1 / 2}=\|\rho\|_{H^{1}(0,2 \pi)} .
$$

The space $W$ does not seem to be very suitable to our problem as the measure $d v$ induces a linear grow in time of the norm due to the expansion of the front. However, it is convenient for energy estimates as we shall see below. In those spaces, the coordinate system developed in the first section is still valid. More precisely, we have the following lemma:

Lemma 2.3. There exist positive constants $R_{1}^{\prime}, \delta_{1}^{\prime}, K^{\prime}$ such that for any $R \geqslant R_{1}^{\prime}$ and any $\xi \in W$ with $\|\xi\|_{W} \leqslant \delta_{1}^{\prime}$, there exists a unique pair $(v, \rho) \in W \times Z$ with

(i) $\|v\|_{W}+\|\rho\|_{Z} \leqslant K^{\prime}\|\xi\|_{W}$,

(ii) $w(r-R, r)+\xi(r, \theta)=w(r-R-\rho(\theta), r)+v(r, \theta)$ for all $(r, \theta) \in \bar{\Omega}$,

(iii) $\int_{0}^{\infty} v(r, \theta) \psi(r-R-\rho(\theta), r) \mathrm{d} r=0$ for any $\theta \in[0,2 \pi)$.

Proof. The proof is very similar to the one of Lemma 1.1 and we may omit it.

Using Lemma 2.3, assuming the solution $u(x, t)$ is close to a travelling wave, we have for any $t \geqslant 0, \theta \in[0,2 \pi)$, and some $R \geqslant 0$,

$$
\begin{aligned}
& u(r, \theta, t)=w(r-s(\theta, t), r)+v(r, \theta, t), \quad r \geqslant 0, \\
& s(\theta, t)=R+c t-\frac{1}{c} \log \left(\frac{R+c t}{R}\right)+\rho(\theta, t), \\
& \int_{0}^{\infty} v(r, \theta, t) \psi(r-s(\theta, t), r) \mathrm{d} r=0 .
\end{aligned}
$$

Note that according to Jones [8], the solution $u(r, \theta, t)$ is close to a travelling wave in every radial direction of $\mathbf{R}^{2}$. Therefore, in (25), $v$ is transversal to $\psi(r-s(\theta, t), r)$ for all $\theta \in[0,2 \pi)$.

Then, we get two new evolution equations. The one satisfied by $v$ is obtained by equations (1) and (24):

$$
\begin{aligned}
v_{t}(r, \theta, t)= & \Delta v(r, \theta, t)+F^{\prime}(w(r-s(\theta, t), r)) v(r, \theta, t)+N+S \\
& +w_{y}(r-s(\theta, t), r) \rho_{t}(\theta, t)-\frac{1}{r^{2}} \partial_{\theta}\left(w_{y}(r-s(\theta, t), r) \rho_{\theta}(\theta, t)\right), \\
v(r, \theta, 0)= & v_{0}(r, \theta),
\end{aligned}
$$

where

$$
\begin{aligned}
& \Delta=\partial_{r}^{2}+\frac{1}{r} \partial_{r}+\frac{1}{r^{2}} \partial_{\theta}^{2}, \\
& N=F(w+v)-F(w)-F^{\prime}(w) v, \\
& S=\left(\frac{1}{r}-\frac{1}{R+c t}\right) w_{y}+\left(w_{r r}+2 w_{r y}+\frac{1}{r} w_{r}\right)+w_{y y}+c w_{y}+F(w) .
\end{aligned}
$$

Differentiating Eq. (25) with respect to $t$ and integrating by parts, we get as in the first section, the equation satisfied by $\rho$ :

$$
\begin{aligned}
& \rho_{t}(\theta, t) \lambda(\infty, \theta, t)=-\int_{0}^{\infty} g(r, \theta, t) \mathrm{d} r \\
& \rho(\theta, 0)=\rho_{0}(\theta),
\end{aligned}
$$


where

$$
\begin{aligned}
& \lambda(r, \theta, t)=\int_{0}^{r}\left(\psi(z-s(\theta, t), z) w_{y}-\psi_{y} v\right) \mathrm{d} z, \\
& g(z, \theta, t)=g_{1}(z, \theta, t)+g_{2}(z, \theta, t), \\
& g_{1}(z, \theta, t)=v \Lambda+\psi(z-s(\theta, t), z)(N+S), \\
& g_{2}(z, \theta, t)=-\frac{1}{z^{2}} \psi \partial_{\theta}\left(w_{y} \rho_{\theta}\right)+\frac{1}{z^{2}} \psi v_{\theta \theta}, \\
& \Lambda(z, \theta, t)=\left(\frac{1}{R+c t}-\frac{1}{z}\right) \psi_{y}+\frac{1}{z^{2}} \psi+\left(\psi_{r r}+2 \psi_{r y}-\frac{1}{z} \psi_{r}\right)+\left(\psi_{y y}-c \psi_{y}+F^{\prime}(w) \psi\right) .
\end{aligned}
$$

As in the first section, we consider the initial value problem for Eqs. (26), (27).

Lemma 2.4. There exist $R_{0}>0, \varepsilon_{0}>0$ and $T>0$ such that, for any $R \geqslant R_{0}$ and for all initial data $\left(v_{0}, \rho_{0}\right) \in W \times Z$ with $\left\|v_{0}\right\|_{W} \leqslant \varepsilon_{0}$ and $\left\|\rho_{0}\right\|_{Z} \leqslant \varepsilon_{0}$, the integral equations corresponding to (26), (27) have a unique solution $(v, \rho) \in C^{0}([0, T], W \times Z)$. In addition, $(v, \rho) \in C^{1}((0, T], W) \times C^{1}((0, T], Z)$, and equations (26), (27) are satisfied for $0<t \leqslant T$.

Proof. Define $\varepsilon=\delta_{1}^{\prime}$ and let $\delta$ be as in Corollary 2.2. Choose $0<\varepsilon_{0} \leqslant \delta\left(1+c_{0} \mathrm{e}^{-\gamma_{1} R_{0}}\right)^{-1}$ for some fixed $R_{0}>0$ large enough. Let $\left(v_{0}, \rho_{0}\right) \in W \times Z$ such that $\left\|v_{0}\right\|_{W} \leqslant \varepsilon_{0}$ and $\left\|\rho_{0}\right\|_{Z} \leqslant \varepsilon_{0}$. Finally, define $u_{0}(r, \theta)=$ $w\left(r-R-\rho_{0}(\theta), r\right)+v_{0}(r, \theta)$. Then, $u_{0} \in H^{1}\left(\mathbf{R}^{2}\right)$ and it is a standard result that there exists a unique solution $u(x, t) \in C^{0}\left([0, T], H^{1}\left(\mathbf{R}^{2}\right)\right) \cap C^{1}\left((0, T], H^{1}\left(\mathbf{R}^{2}\right)\right)$ to Eq. (1) with initial data $u_{0}$. According to Corollary 2.2, $u(x, t)$ stay close to a travelling wave in the $L^{\infty}$-norm for all $t>0$. By energy estimates, we show in Sections 2.2.1 and 2.2.2 that this is also the case in the $H^{1}$ norm. Thus, Lemma 2.3 is still valid and there exists a unique pair $(v, \rho) \in W \times Z$ such that (24), (9), (25) hold and $(v, \rho)$ satisfy Eqs. (26), (27).

These two equations are very similar to those found in the first section. We choose here to deal with energy estimates. We study the behaviour of $\|v(t)\|_{W}$ and $\|\rho(t)\|_{Z}$ under the assumption that the initial data are small. We have the following theorem:

Theorem 2.5. There exist positive constants $R_{1}, \varepsilon_{1}, n$ such that if $\left(v_{0}, \rho_{0}\right) \in W \times Z$ satisfy

$$
R^{1 / 2}\left\|v_{0}\right\|_{W}^{2}+\left\|\rho_{0}\right\|_{Z}^{2} \leqslant \varepsilon
$$

for some $R \geqslant R_{1}$ and some $\varepsilon \leqslant \varepsilon_{1}$, then Eqs. (26), (27) have a unique solution $(v, \rho) \in C^{0}([0,+\infty), W \times Z)$ with initial data $\left(v_{0}, \rho_{0}\right)$, and

$$
(R+c t)^{1 / 2}\|v(t)\|_{W}^{2}+\|\rho(t)\|_{Z}^{2} \leqslant n\left(\varepsilon+\frac{1}{R}\right)
$$

for all $t \geqslant 0$.

These estimates will be useful to prove Theorem 2. We now give the proof of the first part of Theorem 2:

Proof of Theorem 2. Let $R_{1}^{\prime}, \delta_{1}^{\prime}, K^{\prime}$ be as in Lemma 2.4, $R_{1}, \varepsilon_{1}, n$ as in Theorem 2.5 and $c_{0}, \gamma_{1}$ as in (14). Choose $R_{0}^{\prime}, \delta_{0}^{\prime}$ and $\eta$ such that:

$$
\begin{aligned}
& R_{0}^{\prime} \geqslant \max \left(R_{1} ; R_{1}^{\prime}\right), \quad \eta=\frac{\sqrt{\varepsilon_{1}}}{2 \sqrt{2} K^{\prime}} \\
& \delta_{0}^{\prime}+c_{0} \mathrm{e}^{-\gamma_{1} R_{0}^{\prime}} \leqslant \min \left(\delta_{1}^{\prime} ; 2 \eta\right), \quad\left(R_{0}^{\prime}\right)^{1 / 4} c_{0} \mathrm{e}^{-\gamma_{1} R_{0}^{\prime}} \leqslant \eta .
\end{aligned}
$$


Let now $u_{0} \in H^{1}\left(\mathbf{R}^{2}\right)$ such that $\left\|u_{0}(x)-w_{0}(|x|-R)\right\|_{H^{1}\left(\mathbf{R}^{2}\right)} \leqslant \delta$ for some $\delta \leqslant \delta_{0}^{\prime}, R \geqslant R_{0}^{\prime}$ and $R^{1 / 4} \delta \leqslant \eta$. Let $\xi(r, \theta)=u_{0}(r, \theta)-w(r-R, r)$. Then, by (14), $\|\xi\|_{W} \leqslant \delta+c_{0} \mathrm{e}^{-\gamma_{1} R} \leqslant \delta_{1}^{\prime}$ and $R \geqslant R_{1}^{\prime}$. Thus, by Lemma 2.3, there exists a unique pair $\left(v_{0}, \rho_{0}\right) \in W \times Z$ such that

(i) $\left\|v_{0}\right\|_{W}+\left\|\rho_{0}\right\|_{Z} \leqslant K^{\prime}\|\xi\|_{W}$,

(ii) $w(r-R, r)+\xi(r, \theta)=w\left(r-R-\rho_{0}(\theta), r\right)+v_{0}(r, \theta)$ for all $(r, \theta) \in \bar{\Omega}$,

(iii) $\int_{0}^{\infty} v_{0}(r, \theta) \psi\left(r-R-\rho_{0}(\theta), r\right) \mathrm{d} r=0$ for any $\theta \in[0,2 \pi)$.

Then, with the above conditions on $R$ and $\varepsilon$,

$$
R^{1 / 2}\left\|v_{0}\right\|_{W}^{2}+\left\|\rho_{0}\right\|_{Z}^{2} \leqslant \varepsilon_{1}, \quad R \geqslant R_{1} .
$$

Then, by Theorem 2.5, Eqs. (26), (27) have a unique solution $(v, \rho)$ in $C^{0}([0,+\infty), W \times Z)$ and

$$
(R+c t)^{1 / 2}\|v(t)\|_{W}^{2}+\|\rho(t)\|_{Z}^{2} \leqslant n\left(\varepsilon+\frac{1}{R}\right) \text { for all } t \geqslant 0 .
$$

Let $u(r, \theta, t)=w(r-s(\theta, t), r)+v(r, \theta, t)$ where $s(\theta, t)$ is given by (9). Then, by (14), $u$ is a solution of (1) satisfying

$$
\left\|u(r, \theta, t)-w_{0}(r-s(\theta, t))\right\|_{W} \leqslant \frac{c_{0}}{(R+c t)^{1 / 4}} .
$$

This ends the proof of the first part of Theorem 2.

We now prove Theorem 2.5. Therefore, we introduce a few functionals linked with the norms of $v$ and $\rho$ in $W$ and $Z$ respectively.

\subsubsection{Definition of primitive and functionals}

If $T>0$ and $(v, \rho) \in C^{1}((0, T], W \times Z)$ is any solution of (26), (27), we first introduce functionals for the functions $v$ and $\rho$ :

$$
\begin{aligned}
& E_{1}(t)=\frac{1}{2}\|v(t)\|_{L^{2}\left(\mathbf{R}^{2}\right)}^{2}=\frac{1}{2} \int_{0}^{2 \pi} \int_{0}^{\infty} v^{2}(r, \theta, t) r \mathrm{~d} r \mathrm{~d} \theta=\frac{1}{2} \int_{\Omega} v^{2} \mathrm{~d} v, \\
& E_{2}(t)=\frac{1}{2}\|\nabla v(t)\|_{L^{2}\left(\mathbf{R}^{2}\right)}^{2}=\frac{1}{2} \int_{\Omega}\left(v_{r}^{2}+\frac{v_{\theta}^{2}}{r^{2}}\right) \mathrm{d} v, \\
& E_{3}(t)=\frac{1}{2}\|\Delta v(t)\|_{L^{2}\left(\mathbf{R}^{2}\right)}^{2}=\frac{1}{2} \int_{\Omega}\left(v_{r r}+\frac{v_{r}}{r}+\frac{v_{\theta \theta}}{r^{2}}\right)^{2} \mathrm{~d} v, \\
& E_{4}(t)=\frac{1}{2}\|\rho(t)\|_{L^{2}(0,2 \pi)}^{2}=\frac{1}{2} \int_{0}^{2 \pi} \rho^{2}(\theta, t) \mathrm{d} \theta \\
& E_{5}(t)=\frac{1}{2}\left\|\rho_{\theta}(t)\right\|_{L^{2}(0,2 \pi)}^{2}=\frac{1}{2} \int_{0}^{2 \pi} \rho_{\theta}^{2}(\theta, t) \mathrm{d} \theta \\
& E_{6}(t)=\frac{1}{2}\left\|\rho_{\theta \theta}(t)\right\|_{L^{2}(0,2 \pi)}^{2}=\frac{1}{2} \int_{0}^{2 \pi} \rho_{\theta \theta}^{2}(\theta, t) \mathrm{d} \theta .
\end{aligned}
$$


It will be useful to consider also the weighted primitive $V$ of $v$ :

$$
V(r, \theta, t)=\int_{0}^{r} v(z, \theta, t) \psi(z-s(\theta, t), z) \mathrm{d} z=-\int_{r}^{\infty} v(z, \theta, t) \psi(z-s(\theta, t), z) \mathrm{d} z .
$$

Note that $V(0, \theta, t)=V(\infty, \theta, t)=0$ as $v$ is a transversal perturbation for any $\theta \in(0,2 \pi)$, see (25). Under the above assumptions on $v$ and $\rho, V \in C^{1}((0, T], W)$ and it satisfies an evolution equation easily computed by integrations by parts from (26), (27):

$$
V_{t}=V_{r r}-\omega_{1}(r, \theta, t) V_{r}+G_{5}(r, \theta, t)
$$

where

$$
\begin{aligned}
& \omega_{1}(r, \theta, t)=2 \frac{\psi_{0}^{\prime}(r-s(\theta, t))}{\psi_{0}(r-s(\theta, t))}+2 \frac{\chi^{\prime}(r)}{\chi(r)}-\frac{1}{r} \\
& G_{5}(r, \theta, t)=\left(1-\frac{\lambda(r, \theta, t)}{\lambda(\infty, \theta, t)}\right) \int_{0}^{r} g(z, \theta, t) \mathrm{d} z-\frac{\lambda(r, \theta, t)}{\lambda(\infty, \theta, t)} \int_{r}^{\infty} g(z, \theta, t) \mathrm{d} z .
\end{aligned}
$$

We also consider the last functional $E_{0}$ for $V$ :

$$
E_{0}(t)=\frac{1}{2}\|V(t)\|_{L^{2}\left(\mathbf{R}^{2}\right)}^{2}=\frac{1}{2} \int_{\Omega} V^{2}(r, \theta, t) \mathrm{d} \nu .
$$

Note that there exist two positive constants $l_{1}$ and $l_{2}$ such that for any $t \in(0, T)$ (see Appendix B),

$$
l_{1} E_{1}(t) \leqslant E_{0}(t) \leqslant l_{2} E_{1}(t) .
$$

We first give the equations satisfied by these functionals and then find the inequalities involving $E_{0}$ to $E_{6}$ which are useful for the next calculations.

Lemma 2.6. If $T>0$ and $(v, \rho) \in C^{1}((0, T], W \times Z)$ is any solution of (26), (27), then $E_{i} \in C^{1}((0, T])$ for $i=0, \ldots, 6 . E_{0}$ satisfies the equation:

$$
\begin{aligned}
\dot{E}_{0}(t)= & -\int_{\Omega} V_{r}^{2} \mathrm{~d} v+\int_{\Omega}\left(\frac{\psi_{0}^{\prime}(r-s(\theta, t))}{\psi_{0}(r-s(\theta, t))}\right)^{\prime} V^{2} \mathrm{~d} v \\
& +\int_{\Omega} \omega_{2}(r, \theta, t) V^{2} \mathrm{~d} r \mathrm{~d} \theta+\int_{\Omega} V(r, \theta, t) G_{5}(r, \theta, t) \mathrm{d} v,
\end{aligned}
$$

where $\omega_{2}(r, \theta, t)=\psi_{0}^{\prime}(r-s(\theta, t)) / \psi_{0}(r-s(\theta, t))+\chi^{\prime}(r) / \chi(r)+\left(\chi^{\prime}(r) / \chi(r)\right)^{\prime} r$.

Moreover, the functions $E_{1}, E_{2}, E_{4}$ and $E_{5}$ satisfy:

$$
\begin{aligned}
\dot{E}_{1}(t)= & -2 E_{2}+\int_{\Omega} F^{\prime}(w) v^{2} \mathrm{~d} v+\int_{\Omega} v\left(w_{y} \rho_{t}-\frac{1}{r^{2}} \partial_{\theta}\left(w_{y} \rho_{\theta}\right)+N+S\right) \mathrm{d} v, \\
\dot{E}_{2}(t)= & -2 E_{3}-\int_{\Omega} \Delta v\left(F^{\prime}(w) v+w_{y} \rho_{t}-\frac{1}{r^{2}} \partial_{\theta}\left(w_{y} \rho_{\theta}\right)+N+S\right) \mathrm{d} \nu, \\
\dot{E}_{4}(t)= & -\int_{\Omega} \frac{\rho_{\theta}^{2}}{r^{2}} \frac{\psi w_{y}}{\lambda(\infty, \theta, t)} \mathrm{d} r \mathrm{~d} \theta+\int_{\Omega} \frac{\rho \rho_{\theta}^{2}}{r^{2}} \frac{\psi_{y} w_{y}}{\lambda(\infty, \theta, t)} \mathrm{d} r \mathrm{~d} \theta \\
& +\int_{\Omega} \frac{\rho \rho_{\theta}}{r^{2}} \psi w_{y} \frac{\lambda_{\theta}(\infty)}{\lambda^{2}(\infty)} \mathrm{d} r \mathrm{~d} \theta-\int_{\Omega} \frac{\rho}{\lambda(\infty, \theta, t)}\left(g_{1}+\frac{1}{r^{2}} \psi v_{\theta \theta}\right) \mathrm{d} r \mathrm{~d} \theta,
\end{aligned}
$$




$$
\begin{aligned}
\dot{E}_{5}(t)= & -\int_{\Omega} \frac{\rho_{\theta \theta}^{2}}{r^{2}} \frac{\psi w_{y}}{\lambda(\infty, \theta, t)} \mathrm{d} r \mathrm{~d} \theta+\int_{\Omega} \frac{\rho_{\theta \theta} \rho_{\theta}^{2}}{r^{2}} \frac{\psi w_{y y}}{\lambda(\infty, \theta, t)} \mathrm{d} r \mathrm{~d} \theta \\
& +\int_{\Omega} \frac{\rho_{\theta \theta}}{\lambda(\infty, \theta, t)}\left(g_{1}+\frac{1}{r^{2}} \psi v_{\theta \theta}\right) \mathrm{d} r \mathrm{~d} \theta .
\end{aligned}
$$

Proof. Obviously, $\dot{E}_{0}(t)=\int_{\Omega} V V_{t} \mathrm{~d} \nu$. Eq. (28) and integrations by parts yield to the desired expression for $\dot{E}_{0}$. The derivatives with respect to $t$ of $E_{1}$ and $E_{2}$ are more easily computed by analogy with the heat equation in $\mathbf{R}^{2}$ with usual coordinates $x \in \mathbf{R}^{2}$ instead of polar coordinates. As far as the functionals for $\rho$ are concerned, $\dot{E}_{4}$ and $\dot{E}_{5}$ are computed by a few integrations by parts. Note that all the functions depending on $\theta$ are $2 \pi$ periodic. The expressions of $\dot{E}_{4}$ and $\dot{E}_{5}$ have been put in that way to highlight the first terms. Indeed, as we shall see below, $\int_{\Omega}\left(\rho_{\theta}^{2} / r^{2}\right)\left(\psi w_{y} / \lambda(\infty)\right) \mathrm{d} r \mathrm{~d} \theta$ behaves essentially like $E_{5}(t) /(R+c t)^{2}$ and $\int_{\Omega}\left(\rho_{\theta \theta}^{2} / r^{2}\right)\left(\psi w_{y} / \lambda(\infty)\right) \mathrm{d} r \mathrm{~d} \theta$ like $E_{6}(t) /(R+c t)^{2}$. These quantities are going to play an important role in the next energy estimates. Finally, we do not mind about $\dot{E}_{3}$ and $\dot{E}_{6}$ as we are only interested in the $H^{1}$ norms of $v$ and $\rho$.

\subsubsection{Bounds on the functionals and proof of Theorem 2.5}

Proposition 2.7. There exist positive constants $R_{2}, \varepsilon_{2}, k, c_{0}, d, e_{6}$ and $e_{i j}$ for $(i, j) \in\{0, \ldots, 6\}^{2}$ such that if $T>0$ and $(v, \rho) \in C^{0}([0, T], W \times Z)$ is any solution of (26), (27) satisfying for all $t \in[0, T]$,

$$
\|v(t)\|_{W}^{2}+\|\rho(t)\|_{Z}^{2} \leqslant \varepsilon
$$

for some $R \geqslant R_{2}$ and some $\varepsilon \leqslant \varepsilon_{2}$, then the following inequalities hold:

$$
\begin{aligned}
\dot{E}_{0}(t) \leqslant & -\int_{\Omega} \psi^{2} v^{2} \mathrm{~d} v+e_{01} E_{1}+e_{02} E_{2}+\frac{e_{6}}{\sqrt{R+c t}} \frac{E_{6}}{(R+c t)^{2}}+\frac{c_{0}}{(R+c t)^{2}}, \\
\dot{E}_{1}(t) \leqslant & -2 E_{2}+\int_{\Omega} F^{\prime}(w) v^{2} \mathrm{~d} v+e_{11} E_{1}+e_{12} E_{2}+e_{13} E_{3} \\
& +e_{15} \frac{E_{5}}{(R+c t)^{2}}+\frac{e_{6}}{\sqrt{R+c t}} \frac{E_{6}}{(R+c t)^{2}}+\frac{c_{0}}{(R+c t)^{3}}, \\
\dot{E}_{2}(t) \leqslant & -2 E_{3}+\left(e_{21}+(d k)^{2}\right) E_{1}+e_{22} E_{2}+\left(e_{23}+1\right) E_{3} \\
& +e_{25} \frac{E_{5}}{(R+c t)^{2}}+\frac{e_{6}}{\sqrt{R+c t}} \frac{E_{6}}{(R+c t)^{2}}+\frac{c_{0}}{(R+c t)^{3}}, \\
\dot{E}_{4}(t) \leqslant & -d \frac{E_{5}}{(R+c t)^{2}}+e_{41} E_{1}+e_{42} E_{2}+e_{43} E_{3}+e_{45} \frac{E_{5}}{(R+c t)^{2}}+e_{46} \frac{E_{6}}{(R+c t)^{2}}+\frac{c_{0}}{(R+c t)^{2}}, \\
\dot{E}_{5}(t) \leqslant & -d \frac{E_{6}}{(R+c t)^{2}}+e_{51} E_{1}+e_{52} E_{2}+e_{53} E_{3}+\left(e_{56}+\frac{d}{4}\right) \frac{E_{6}}{(R+c t)^{2}} \\
& +\frac{c_{0}}{(R+c t)^{2}}+\frac{2}{d}(R+c t)\left(E_{1}+E_{2}\right)^{2}
\end{aligned}
$$

and

$$
\sup _{x \in \mathbf{R}}\left(F^{\prime}\left(w_{0}(x)\right)-k \psi_{0}^{2}(x)\right) \leqslant-2 .
$$

Moreover, constants $e_{i j}$ can be chosen as small as we want by choosing $R_{2}$ large enough and $\varepsilon_{2}$ small enough.

We prove right now how Theorem 2.5 follows from Proposition 2.7. 
Proof of Theorem 2.5. Let $R_{2}, \varepsilon_{2}, k, c_{0}, d, e_{6}$ and $e_{i j}$ be as in Proposition 2.7, $R_{0}, \varepsilon_{0}$ be as in Lemma 2.4 and $l_{1}, l_{2}$ as in (29). Choose $m>0, R_{1}>0, \varepsilon_{1}>0, l=1 / a>0$ such that

$$
R_{1} \geqslant \max \left(1, R_{0}, R_{2}, a^{2}, a c\right), \quad \varepsilon_{1} \leqslant \min \left(\varepsilon_{0}^{2}, \varepsilon_{2}\right), \quad \frac{\sqrt{\varepsilon_{1}}}{R_{1}^{1 / 4}} \leqslant \varepsilon_{0}, \quad m(d k)^{2} \leqslant \frac{1}{2},
$$

where $a=\max \left(1+k l_{2}, m\right)$ and $b=\min \left(1+k l_{1}, m\right)$. We also request that for any $R \geqslant R_{1}$, and any $0<\varepsilon \leqslant \varepsilon_{1}$, the following inequalities hold for any $t \geqslant 0$ :

$$
\begin{aligned}
& k e_{01}+e_{11}+m\left(e_{21}+(d k)^{2}\right)+e_{41}+e_{51} \leqslant 1, \\
& -2+k e_{02}+e_{12}+m e_{22}+e_{42}+e_{52} \leqslant-1, \\
& -2 m+e_{13}+m\left(e_{23}+1\right)+e_{43}+e_{53} \leqslant-\frac{m}{2}, \\
& -d+e_{15}+m e_{25}+e_{45} \leqslant 0, \\
& -d+k \frac{e_{6}}{\sqrt{R+c t}}+\frac{e_{6}}{\sqrt{R+c t}}+m \frac{e_{6}}{\sqrt{R+c t}}+e_{46}+e_{56}+\frac{d}{4} \leqslant-\frac{d}{2} .
\end{aligned}
$$

This is possible by first choosing $m>0$, then $\varepsilon_{1}$ small enough and $R_{1}$ large enough. Take $R \geqslant R_{1}, \varepsilon \leqslant \varepsilon_{1}$ and $\left(v_{0}, \rho_{0}\right) \in W \times Z$ satisfying

$$
R^{1 / 2}\left\|v_{0}\right\|_{W}^{2}+\left\|\rho_{0}\right\|_{Z}^{2} \leqslant \varepsilon .
$$

By Lemma 2.4, let $(v, \rho) \in C^{0}\left(\left[0, T^{*}\right), W \times Z\right)$ be the maximal solution of (26), (27) with initial data $\left(v_{0}, \rho_{0}\right)$. Define, for some $n \in \mathbf{N}^{*}$,

$$
\begin{array}{r}
T=\sup \left\{\widetilde{T} \in\left[0, T^{*}\right) \mid(R+c t)^{1 / 2}\|v(t)\|_{W}^{2}+\|\rho(t)\|_{Z}^{2} \leqslant n\left(\varepsilon+\frac{1}{R}\right)\right. \\
\left.\qquad \quad \text { and } \int_{0}^{t}(R+c s) \mathcal{U}^{2}(s) \mathrm{d} s \leqslant 2\left(\varepsilon+\frac{1}{R}\right) \text { for } 0 \leqslant t \leqslant \widetilde{T}\right\},
\end{array}
$$

where

$$
\mathcal{U}(t)=k E_{0}+E_{1}+m E_{2} \quad \text { and } \quad b\left(E_{1}+E_{2}\right) \leqslant \mathcal{U}(t) \leqslant a\left(E_{1}+E_{2}\right) .
$$

We also give some conditions on $n$ : we assume that

$$
\begin{aligned}
& \frac{a}{b}+\left(\frac{2(k+1+m) e_{6}}{d b}(1+\sqrt{2})+1\right)\left(a+\frac{4}{d b^{2}}\right) \leqslant n-1, \\
& \left(\frac{2(k+1+m) e_{6}}{d b}(1+\sqrt{2})+1\right)\left(\frac{\bar{c}}{c}+\frac{4}{d b^{2}}\right)+\frac{\bar{c}}{2 b}+\frac{\bar{c} \sqrt{2}}{b l} \leqslant n-1, \\
& \frac{a^{2} \varepsilon_{1}}{l}+\frac{2 a n}{l}\left(\frac{2(k+1+m) e_{6} \tilde{\varepsilon}_{1}}{d}+\frac{2 \bar{c}}{c R_{1}^{1 / 2}}\right) \leqslant 1, \\
& n\left(\varepsilon_{1}+\frac{1}{R_{1}}\right) \leqslant \varepsilon_{2},
\end{aligned}
$$

where $\tilde{\varepsilon}_{1}=a \varepsilon_{1}+\bar{c} /\left(c R_{1}\right)+4 /\left(d b^{2}\right)\left(\varepsilon_{1}+1 / R_{1}\right)$ and $\bar{c}$ is definied by (40), (43) and (44). This is possible by first choosing $n$ large enough such that the first two inequalities are valid and finally $\varepsilon_{1}$ small enough and $R_{1}$ large enough such that the last two inequalities hold.

By continuity of $v$ and $\rho$, it is clear that $T>0$. We claim that $T=T^{*}$, which also implies $T=T^{*}=+\infty$. Then, the inequalities satisfied by $v$ and $\rho$ are true for all $t \geqslant 0$ and Theorem 2.5 follows immediately. Indeed, if 
$T<T^{*}$, it follows from Proposition 2.7 and inequality (39) that for $t \in[0, T]$, inequalities (34) are satisfied. To get a contradiction on the definition of $T$, we must judiciously bound the expressions $(R+c t)^{1 / 2}\|v(t)\|_{W}^{2}+\|\rho(t)\|_{Z}^{2}$ and $\int_{0}^{t}(R+c s) \mathcal{U}^{2}(s) \mathrm{d} s$. Therefore, define

$$
\mathcal{E}(t)=k E_{0}+E_{1}+m E_{2}+E_{4}+E_{5}=\mathcal{U}(t)+E_{4}+E_{5} .
$$

Using (34) and (36), there exists $\bar{c}>0$ such that

$$
\dot{\mathcal{E}}(t) \leqslant-E_{1}-E_{2}-\frac{m}{2} E_{3}(t)-\frac{d E_{6}(t)}{2(R+c t)^{2}}+\frac{\bar{c}}{(R+c t)^{2}}+\frac{2}{d}(R+c t)\left(E_{1}+E_{2}\right)^{2} .
$$

Integrating this inequality between 0 and $t \leqslant T$, we get

$$
\begin{aligned}
& \mathcal{E}(t)+\int_{0}^{t}\left(E_{1}+E_{2}\right)(s) \mathrm{d} s+\int_{0}^{t} \frac{m}{2} E_{3}(s) \mathrm{d} s+\int_{0}^{t} \frac{d E_{6}(s)}{2(R+c s)^{2}} \mathrm{~d} s \\
& \quad \leqslant \mathcal{E}(0)+\int_{0}^{t} \frac{\bar{c}}{(R+c s)^{2}} \mathrm{~d} s+\int_{0}^{t} \frac{2}{d}(R+c s)\left(E_{1}+E_{2}\right)^{2}(s) \mathrm{d} s \leqslant \tilde{\varepsilon},
\end{aligned}
$$

where $\tilde{\varepsilon}=a \varepsilon+\bar{c} /(c R)+4 /\left(d b^{2}\right)(\varepsilon+1 / R)$. Moreover, we also get from inequalities (34) that

$$
\dot{\mathcal{U}}(t) \leqslant-E_{1}(t)-E_{2}(t)+f(t) \leqslant-l \mathcal{U}(t)+f(t),
$$

where

$$
f(t)=\frac{(k+1+m) e_{6}}{\sqrt{R+c t}} \frac{E_{6}(t)}{(R+c t)^{2}}+\frac{\left(e_{15}+m e_{25}\right) E_{5}(t)}{(R+c t)^{2}}+\frac{c_{0}}{(R+c t)^{2}} .
$$

Then, $\mathcal{U}(t) \leqslant \mathcal{U}(0) \mathrm{e}^{-l t}+\int_{0}^{t} \mathrm{e}^{-l(t-s)} f(s) \mathrm{d} s$. Finally,

$$
E_{1}(t)+E_{2}(t) \leqslant \frac{a \varepsilon}{b \sqrt{R}} \mathrm{e}^{-l t}+\int_{0}^{t} \frac{f(s)}{b} \mathrm{e}^{-l(t-s)} \mathrm{d} s .
$$

To evaluate this last integral, we cut it into two parts and use inequality (41) and the fact that $E_{5}(t) \leqslant n(\varepsilon+1 / R) \leqslant$ $\varepsilon_{2}$ :

$$
\begin{aligned}
\int_{0}^{t / 2} \mathrm{e}^{-l(t-s)} f(s) d s & \leqslant \mathrm{e}^{-l t / 2}\left(\frac{2(k+1+m) e_{6} \tilde{\varepsilon}}{d \sqrt{R}}+\frac{c_{0} t}{2 R^{2}}\right) \\
\int_{t / 2}^{t} \mathrm{e}^{-l(t-s)} f(s) \mathrm{d} s & \leqslant \frac{e_{6}(k+1+m)}{\sqrt{R+c t / 2}} \int_{t / 2}^{t} \frac{E_{6}(s)}{(R+c s)^{2}} \mathrm{~d} s+\frac{c_{0}}{l(R+c t / 2)^{2}} \\
& \leqslant \frac{2(k+1+m) e_{6} \tilde{\varepsilon}}{d \sqrt{R+c t / 2}}+\frac{c_{0}}{l(R+c t / 2)^{2}} .
\end{aligned}
$$

Finally, using (36), (37), (41) and the above inequalities, there exists $\bar{c}>0$ such that

$$
\begin{aligned}
& (R+c t)^{1 / 2}\left(E_{1}+E_{2}\right)(t)+E_{4}(t)+E_{5}(t) \\
& \quad \leqslant \frac{a \varepsilon}{b}+\frac{2(k+1+m) e_{6} \tilde{\varepsilon}}{d b}+\frac{\bar{c}}{2 b R^{3 / 2}}+\frac{\sqrt{2}}{b}\left(\frac{2(k+1+m) e_{6} \tilde{\varepsilon}}{d}+\frac{\bar{c}}{l R^{3 / 2}}\right)+\tilde{\varepsilon} \\
& \quad \leqslant(n-1)\left(\varepsilon+\frac{1}{R}\right) .
\end{aligned}
$$


We now want to evaluate the integral $\int_{0}^{t}(R+c s) \mathcal{U}^{2}(s) \mathrm{d} s$. Therefore, define

$$
\mathcal{G}(t)=(R+c t) \mathcal{U}^{2}(t) .
$$

Then, using (42) and $R_{1} \geqslant a c$,

$$
\frac{\mathrm{d} \mathcal{G}}{\mathrm{d} t} \leqslant-l \mathcal{G}+2 a n\left(\varepsilon+\frac{1}{R}\right)\left(\frac{(k+1+m) e_{6} E_{6}(t)}{(R+c t)^{2}}+\frac{c_{0}}{(R+c t)^{3 / 2}}\right) .
$$

By Gronwall's lemma, we get a bound on $\mathcal{G}$ and by integrating between 0 and $t$,

$$
\int_{0}^{t} \mathcal{G}(s) \mathrm{d} s \leqslant \frac{a^{2} \varepsilon^{2}}{l}+2 a n\left(\varepsilon+\frac{1}{R}\right) \int_{0}^{t} \int_{0}^{s} \mathrm{e}^{-l(s-\tau)}\left(\frac{(k+1+m) e_{6} E_{6}(\tau)}{(R+c \tau)^{2}}+\frac{c_{0}}{(R+c \tau)^{3 / 2}}\right) \mathrm{d} \tau \mathrm{d} s .
$$

Finally, by Fubini's theorem, (41) and (38), there exists $\bar{c}>0$ such that

$$
\int_{0}^{t}(R+c s) \mathcal{U}^{2}(s) \mathrm{d} s \leqslant \frac{a^{2} \varepsilon^{2}}{l}+\frac{2 a n}{l}\left(\varepsilon+\frac{1}{R}\right)\left(\frac{2(k+1+m) e_{6} \tilde{\varepsilon}}{d}+\frac{2 \bar{c}}{c R^{1 / 2}}\right) \leqslant\left(\varepsilon+\frac{1}{R}\right) .
$$

Then, by (43) and (44), we get for any $\varepsilon \leqslant \varepsilon_{1}$ and any $R \geqslant R_{1}$,

$$
\begin{aligned}
& (R+c t)^{1 / 2}\left(E_{1}+E_{2}\right)+E_{4}+E_{5} \leqslant(n-1)\left(\varepsilon+\frac{1}{R}\right), \\
& \int_{0}^{t}(R+c s) \mathcal{U}^{2} \mathrm{~d} s \leqslant\left(\varepsilon+\frac{1}{R}\right)
\end{aligned}
$$

for all $0 \leqslant t \leqslant T$. This contradicts the definition of $T$ and concludes the proof.

\subsubsection{Proof of Proposition 2.7}

The proof of Proposition 2.7 is technical and we need a few intermediate lemmas to prove inequalities (34). We only use a few fundamental ideas: Cauchy-Schwartz' inequality, Jensen's inequality, Schur's lemma and the fact that $\psi_{0}(r-R-c t)$ and $\phi_{0}(r-R-c t)$ are localized around $r=R+c t$. We encourage the reader to refer to Appendix B where we explain in detail the way those fundamental ideas are used in the following lemmas. For the whole Section 2.2.3, we call $(H)$ the following assumptions:

Fix $\varepsilon, R, T$ positive constants.

Let $(v, \rho) \in C^{0}([0, T], W \times Z)$ be any solution of (26), (27) satisfying

$$
\|v(t)\|_{W}^{2}+\|\rho(t)\|_{Z}^{2} \leqslant \varepsilon, \quad t \in[0, T]
$$

In the following six lemmas, we prove that inequalities (34) follow from Eqs. (30)-(33) $-\dot{E}_{0}$ to $\dot{E}_{6}$ and inequality (45).

Lemma 2.8. Under assumptions $(\mathrm{H})$, there exist positive constants $R_{2}, \varepsilon_{2}, c_{0}$ such that for any $t \in(0, T]$ and any $R \geqslant R_{2}, \varepsilon \leqslant \varepsilon_{2}$,

$$
\left\|\rho_{t}\right\|_{L^{2}(0,2 \pi)} \leqslant c_{0}\left(\frac{E_{6}^{1 / 2}}{(R+c t)^{2}}+\frac{E_{5}^{3 / 4} E_{6}^{1 / 4}}{(R+c t)^{2}}+A+B\right)
$$

where 


$$
\begin{aligned}
A= & \left(\frac{E_{6}}{(R+c t)^{2}}\right)^{1 / 2}\left[\frac{E_{1}}{(R+c t)^{2}}+\frac{\left(E_{1}+E_{2}\right)^{1 / 2} E_{5}^{1 / 4}}{(R+c t)^{5 / 4}}+\frac{\left(E_{1} E_{2}\right)^{1 / 4}}{R+c t}+\frac{\left(E_{1} E_{5}\right)^{1 / 2}}{(R+c t)^{3 / 2}}\right] \\
& +\frac{E_{3}^{1 / 2}}{(R+c t)^{3 / 2}}+\left(\frac{E_{6}}{(R+c t)^{2}}\right)^{1 / 4} \frac{E_{2}^{1 / 2} E_{5}^{1 / 4}}{R+c t}, \\
B= & \frac{2 E_{1}^{1 / 2}}{(R+c t)^{5 / 2}}+\frac{E_{1}+E_{2}}{\sqrt{R+c t}}+\frac{1}{(R+c t)^{2}} .
\end{aligned}
$$

Proof. $\rho$ is a solution of Eq. (27) and we want to bound the $L^{2}$ norm of $\rho_{t}$. Therefore, we need to bound $\lambda(\infty, \theta, t)$ from below and $\left|\int_{0}^{\infty} g(r, \theta, t) \mathrm{d} r\right|$ from above. Using Jensen's and Cauchy-Schwartz' inequalities and the Sobolev's embedding $H^{1}\left(\mathbf{R}^{2}\right) \hookrightarrow L^{4}\left(\mathbf{R}^{2}\right)$, we first have

$$
\begin{aligned}
& \sup _{\theta \in(0,2 \pi)}\left|\int_{0}^{\infty} \psi_{y} v \mathrm{~d} r\right|^{2} \leqslant \sup _{\theta \in(0,2 \pi)} \int_{0}^{\infty}\left|\psi_{y} v^{2}\right| \mathrm{d} r \quad \text { (Jensen) } \\
& \leqslant \int_{0}^{2 \pi} \int_{0}^{\infty}\left|v^{2} \psi_{y}\right| \mathrm{d} r \mathrm{~d} \theta+\int_{0}^{2 \pi} \int_{0}^{\infty}\left|2 v v_{\theta} \psi_{y}\right| \mathrm{d} r \mathrm{~d} \theta+\int_{0}^{2 \pi} \int_{0}^{\infty}\left|v^{2} \rho_{\theta} \psi_{y y}\right| \mathrm{d} r \mathrm{~d} \theta \\
& \leqslant c_{0}\left(\frac{E_{1}}{R+c t}+\left(E_{1} E_{2}\right)^{1 / 2}+E_{5}^{1 / 2} \frac{\left(E_{1}+E_{2}\right)}{(R+c t)^{1 / 2}}\right) \leqslant c_{0} \varepsilon
\end{aligned}
$$

as for any function $f$ such that $\int_{0}^{2 \pi} f(\theta) \mathrm{d} \theta=0, \sup |f| \leqslant \int_{0}^{2 \pi}\left|f_{\theta}\right| \mathrm{d} \theta$.

As $\lambda(\infty, \theta, t)=\int_{0}^{\infty} \psi w_{y} \mathrm{~d} r-\int_{0}^{\infty} \psi_{y} v \mathrm{~d} r$ and $\int_{0}^{\infty} \psi w_{y} \mathrm{~d} r=1-\mathrm{O}\left(\mathrm{e}^{-(R+c t)}\right)$, we have

$$
1-c_{0}\left(\varepsilon^{1 / 2}+\mathrm{e}^{-R}\right) \leqslant \lambda(\infty, \theta, t)
$$

for any $\theta \in(0,2 \pi)$ and any $t>0$. Then, for convenient $\varepsilon_{2}$ and $R_{2}, \lambda(\infty, \theta, t)^{-1} \leqslant 2$ for any $\theta \in(0,2 \pi), t>0$, $R \geqslant R_{2}$ and $\varepsilon \leqslant \varepsilon_{2}$.

Moreover, using Schur's lemma (see Appendix B), we have

$$
\left\|\rho_{\theta \theta} \int_{0}^{\infty} \frac{\psi w_{y}}{r^{2} \lambda(\infty, \theta, t)} \mathrm{d} r\right\|_{L^{2}(0,2 \pi)} \leqslant \frac{c_{0} E_{6}^{1 / 2}}{(R+c t)^{2}}
$$

and

$$
\left\|\rho_{\theta}^{2} \int_{0}^{\infty} \frac{\psi w_{y y}}{r^{2} \lambda(\infty, \theta, t)} \mathrm{d} r\right\|_{L^{2}(0,2 \pi)} \leqslant \frac{c_{0}}{(R+c t)^{2}}\left\|\rho_{\theta}\right\|_{L^{2}(0,2 \pi)}\left\|\rho_{\theta}\right\|_{L^{\infty}(0,2 \pi)} \leqslant \frac{c_{0} E_{5}^{3 / 4} E_{6}^{1 / 4}}{(R+c t)^{2}}
$$

as $\left\|\rho_{\theta}\right\|_{L^{\infty}(0,2 \pi)} \leqslant\left(E_{5} E_{6}\right)^{1 / 4}$. To bound the norm of $\int_{0}^{\infty}\left(\psi v_{\theta \theta} /\left(r^{2} \lambda(\infty)\right)\right) \mathrm{d} r$, we introduce the difference $1 / r^{2}-1 /(R+c t)^{2}$ :

$$
\int_{0}^{\infty} \frac{\psi v_{\theta \theta}}{r^{2} \lambda(\infty, \theta, t)} \mathrm{d} r=\int_{0}^{\infty}\left(\frac{1}{(R+c t)^{2}}-\frac{1}{r^{2}}\right) \frac{\psi v_{\theta \theta}}{\lambda(\infty)} \mathrm{d} r+\frac{1}{(R+c t)^{2}} \int_{0}^{\infty} \frac{\psi v_{\theta \theta}}{\lambda(\infty)} \mathrm{d} r .
$$


The first term is bounded in the $L^{2}(0,2 \pi)$ norm by $E_{3}^{1 / 2} /(R+c t)^{3 / 2}$. For the second one, we write $\int_{0}^{\infty} \psi v_{\theta \theta} \mathrm{d} r$ with derivatives of $\rho$ and $v$ by derivating twice identity (11) with respect to $\theta$ :

$$
\int_{0}^{\infty} \psi v_{\theta \theta} \mathrm{d} r=\rho_{\theta \theta} \int_{0}^{\infty} \psi_{y} v \mathrm{~d} r-\rho_{\theta}^{2} \int_{0}^{\infty} \psi_{y y} v \mathrm{~d} r+2 \rho_{\theta} \int_{0}^{\infty} \psi_{y} v_{\theta} \mathrm{d} r
$$

Finally, by Jensen's and Cauchy-Schwartz' inequalities, Schur's lemma and the Sobolev's embedding $H^{1}\left(\mathbf{R}^{2}\right) \hookrightarrow$ $L^{4}\left(\mathbf{R}^{2}\right)$, we get

$$
\begin{aligned}
\left\|\int_{0}^{\infty} \psi v_{\theta \theta} \mathrm{d} r\right\|_{L^{2}(0,2 \pi)} \leqslant & c_{0}\left(\int_{0}^{2 \pi} \int_{0}^{\infty} \rho_{\theta \theta}^{2} \psi_{y} v^{2} \mathrm{~d} r \mathrm{~d} \theta\right)^{1 / 2} \\
& +c_{0}\left(\int_{0}^{2 \pi} \int_{0}^{\infty} \rho_{\theta}^{4} \psi_{y y} v^{2} \mathrm{~d} r \mathrm{~d} \theta\right)^{1 / 2}+c_{0}\left(\iint_{0}^{2 \pi} \rho_{0}^{\infty} \rho_{\theta} v_{\theta}^{2} \mathrm{~d} r \mathrm{~d} \theta\right)^{1 / 2} \\
\leqslant & c_{0}\left\|\rho_{\theta \theta}\right\|_{L^{2}(0,2 \pi)}\left\|\int_{0}^{\infty} \psi_{y} v^{2} \mathrm{~d} r\right\|_{L^{\infty}(0,2 \pi)}^{1 / 2}+c_{0}\left\|\rho_{\theta}\right\|_{L^{\infty}(0,2 \pi)}^{2}\left(\int_{0}^{2 \pi} \int_{0}^{\infty} \frac{v^{2}}{r} \psi_{y y} \mathrm{~d} v\right)^{1 / 2} \\
& +c_{0}\left\|\rho_{\theta}\right\|_{L^{\infty}(0,2 \pi)}\left(\int_{0}^{2 \pi} \int_{0}^{\infty} \frac{v_{\theta}^{2}}{r^{2}} \psi_{y} r \mathrm{~d} v\right)^{1 / 2} \\
\leqslant & c_{0} E_{6}^{1 / 2}\left(\frac{E_{1}}{R+c t}+\left(E_{1} E_{2}\right)^{1 / 4}+E_{5}^{1 / 4} \frac{\left(E_{1}+E_{2}\right)^{1 / 2}}{(R+c t)^{1 / 4}}\right) \\
& +c_{0}\left(\frac{E_{1} E_{5} E_{6}}{R+c t}\right)^{1 / 2}+c_{0}\left(E_{5} E_{6}\right)^{1 / 4} \sqrt{R+c t} E_{2}^{1 / 2} .
\end{aligned}
$$

Then,

$$
\left\|\int_{0}^{\infty} \frac{\psi v_{\theta \theta}}{r^{2} \lambda(\infty, \theta, t)} \mathrm{d} r\right\|_{L^{2}(0,2 \pi)} \leqslant c_{0} A .
$$

The last term $\int_{0}^{\infty} v \Lambda+\psi(N+S) \mathrm{d} r$ is bounded by Jensen's inequality, Schur's lemma (see Appendix B) and the Sobolev's embedding $H^{1}\left(\mathbf{R}^{2}\right) \hookrightarrow L^{4}\left(\mathbf{R}^{2}\right)$. Then, $\left\|\int_{0}^{\infty} v \Lambda+\psi(N+S) \mathrm{d} r\right\|_{L^{2}(0,2 \pi)} \leqslant c_{0} B$. Notice that as $H^{1}\left(\mathbf{R}^{2}\right)$ is not an algebra, we need some more assumptions to bound the norm of $N$. We assumed in the introduction that every solution of $u_{t}=F(u)$ is uniformly bounded in time. Therefore, $v$ is bounded and Taylor's theorem and Sobolev's embedding enable us to bound $\|N\|_{L^{2}\left(\mathbf{R}^{2}\right)}$. This concludes the proof of Lemma 2.8 .

Lemma 2.9. Under assumptions $(\mathrm{H})$, there exist positive constants $c_{0}, R_{2}, \varepsilon_{2}$ such that for any $t \in[0, T]$ and any $R \geqslant R_{2}, \varepsilon \leqslant \varepsilon_{2}$,

$$
\dot{E}_{0}(t) \leqslant-\int_{\Omega} \psi^{2} v^{2} \mathrm{~d} v+c_{0}\left(\frac{E_{1}}{R+c t}+E_{0}^{1 / 2} B+C\right),
$$

where 


$$
\begin{aligned}
C= & \frac{E_{2}}{R+c t}+\left(\frac{E_{6}}{(R+c t)^{2}}\right)^{1 / 4}\left(\frac{\left(E_{1} E_{2}\right)^{1 / 2} E_{5}^{1 / 4}}{\sqrt{R+c t}}+\frac{\left(E_{0} E_{2}\right)^{1 / 2} E_{5}^{1 / 4}}{\sqrt{R+c t}}\right) \\
& +\left(\frac{E_{6}}{(R+c t)^{2}}\right)^{1 / 2}\left(\frac{\left(E_{0} E_{5}\right)^{1 / 2}}{R+c t}+\frac{\left(E_{1} E_{5}\right)^{1 / 2}}{R+c t}\right)+\frac{\left(E_{2} E_{5}\right)^{1 / 2}}{R+c t} .
\end{aligned}
$$

Consequently, there exist positive constants $e_{01}, e_{02}, e_{6}$ such that

$$
\dot{E}_{0}(t) \leqslant-\int_{\Omega} \psi^{2} v^{2} \mathrm{~d} v+e_{01} E_{1}+e_{02} E_{2}+\frac{e_{6}}{\sqrt{R+c t}} \frac{E_{6}}{(R+c t)^{2}}+\frac{c_{0}}{(R+c t)^{2}},
$$

where $e_{01}$ and $e_{02}$ can be chosen small with appropriate $R_{2}$ and $\varepsilon_{2}$.

Proof. We know that $V_{r}=\psi v$; by Appendix C, we have $\left(\psi_{0}^{\prime} / \psi_{0}\right)^{\prime}=\left(\phi_{0}^{\prime} / \phi_{0}\right)^{\prime}<0$ and there exists some constant $c_{0}>0$ such that $\left|\omega_{2}\right|<c_{0}$. Then, by Eq. (30), the only difficulty in $\dot{E}_{0}$ comes from $\int_{\Omega} V G_{5} \mathrm{~d} \nu$. If $r \ll R+c t$, the main term in $G_{5}$ is $\int_{0}^{r} g \mathrm{~d} z$ and if $r \gg R+c t, \int_{r}^{\infty} g \mathrm{~d} z$. We bound separately the term with $g_{1}$ and the one with $g_{2}$.

The term with $g_{1}$ is bounded by $E_{0}^{1 / 2} B$ as in Lemma 2.8. The term with $g_{2}$ is bounded after one integration by parts in $\theta$, Cauchy-Schwartz' and Jensen's inequalities by $C$. Indeed, if $r \ll R+c t$, as

$$
\begin{aligned}
& V_{\theta}=\int_{0}^{r}\left(\psi v_{\theta}-\rho_{\theta} \psi_{y} v\right) \mathrm{d} z \\
& \int_{0}^{R+c t} \int_{0}^{2 \pi} V(r, \theta, t) \int_{0}^{r} g_{2}(z, \theta, t) \mathrm{d} z \mathrm{~d} v=-\int_{0}^{R+c t} \int_{0}^{2 \pi} \frac{1}{r^{2}}\left(\int_{0}^{r} \psi v_{\theta} d z\right)^{2} \mathrm{~d} v \\
& +\int_{0}^{R+c t} \int_{0}^{2 \pi}\left(\int_{0}^{r} \psi v_{\theta} \mathrm{d} z\right)\left(\int_{0}^{r} \psi v_{\theta}\left(\frac{1}{r^{2}}-\frac{1}{z^{2}}\right) \mathrm{d} z\right) \mathrm{d} v+\int_{0}^{R+c t} \int_{0}^{2 \pi} \rho_{\theta}\left(\int_{0}^{r} \psi_{y} v \mathrm{~d} z\right)\left(\int_{0}^{r} \psi \frac{v_{\theta}}{z^{2}} \mathrm{~d} z\right) \mathrm{d} \nu \\
& +\int_{0}^{R+c t} \int_{0}^{2 \pi} \rho_{\theta} V\left(\int_{0}^{r} \psi_{y} \frac{v_{\theta}}{z^{2}} \mathrm{~d} z\right) \mathrm{d} \nu-\int_{0}^{R+c t} \int_{0}^{2 \pi} \rho_{\theta}^{2} V\left(\int_{0}^{r} \frac{\psi_{y} w_{y}}{z^{2}} \mathrm{~d} z\right) \mathrm{d} \nu \\
& +\int_{0}^{R+c t} \int_{0}^{2 \pi} \rho_{\theta}\left(\int_{0}^{r} \frac{\psi w_{y}}{z^{2}} \mathrm{~d} z\right)\left(\int_{0}^{r} \psi v_{\theta} \mathrm{d} z\right) \mathrm{d} \nu-\int_{0}^{R+c t} \int_{0}^{2 \pi} \rho_{\theta}^{2}\left(\int_{0}^{r} \frac{\psi w_{y}}{z^{2}} \mathrm{~d} z\right)\left(\int \psi_{y} v \mathrm{~d} z\right) \mathrm{d} \nu .
\end{aligned}
$$

Notice that the first term is negative and can be omitted. The following terms can be treated as described before. Inequality (34) for $\dot{E}_{0}$ is easily computed from this result using inequalities such as $a b \leqslant\left(a^{2}+b^{2}\right) / 2$. Then,

$$
\begin{aligned}
& e_{01}=c_{0}\left(\frac{1}{R+c t}+\frac{\sqrt{\varepsilon}}{\sqrt{R+c t}}+\frac{\sqrt{\varepsilon}}{R+c t}\right), \\
& e_{02}=c_{0}\left(\frac{1}{R+c t}+\frac{\sqrt{\varepsilon}}{\sqrt{R+c t}}+\sqrt{\varepsilon}\right), \\
& e_{6}=c_{0}\left(\frac{\sqrt{\varepsilon}}{\sqrt{R+c t}}+\sqrt{\varepsilon}\right) .
\end{aligned}
$$

We easily notice that $e_{01}$ and $e_{02}$ can be chosen very small with appropriate $R_{2}$ and $\varepsilon_{2}$. 
Lemma 2.10. Under assumptions $(\mathrm{H})$, there exist positive constants $c_{0}, R_{2}, \varepsilon_{2}$ such that for any $t \in(0, T]$ and any $R \geqslant R_{2}, \varepsilon \leqslant \varepsilon_{2}$,

$$
\dot{E}_{1}(t) \leqslant-2 E_{2}+\int_{\Omega} F^{\prime}(w) v^{2} \mathrm{~d} v+c_{0}\left(E_{1}^{1 / 2} D+\left(E_{1}+E_{2}\right)^{3 / 2}\right),
$$

where

$$
D=\sqrt{R+c t}\left\|\rho_{t}\right\|_{L^{2}}+\frac{1}{\sqrt{R+c t}}\left(\frac{E_{6}}{(R+c t)^{2}}\right)^{1 / 2}+\frac{E_{5}^{3 / 4}}{R+c t}\left(\frac{E_{6}}{(R+c t)^{2}}\right)^{1 / 4}+\frac{1}{(R+c t)^{3 / 2}} .
$$

Consequently, there exist positive constants $e_{11}, e_{12}, e_{13}, e_{15}$ and $e_{6}$ such that

$$
\begin{aligned}
\dot{E}_{1}(t) \leqslant & -2 E_{2}+\int_{\Omega} F^{\prime}(w) v^{2} \mathrm{~d} v+e_{11} E_{1}+e_{12} E_{2}+e_{13} E_{3} \\
& +e_{15} \frac{E_{5}}{(R+c t)^{2}}+\frac{e_{6}}{\sqrt{R+c t}} \frac{E_{6}}{(R+c t)^{2}}+\frac{c_{0}}{(R+c t)^{3}},
\end{aligned}
$$

where $\left\{e_{1 j}\right\}_{j=1, \ldots, 5}$ can be chosen small with appropriate $R_{2}$ and $\varepsilon_{2}$.

Proof. From Eq. (31), we bound $\dot{E}_{1}(t)$ term by term: $\left\|v \rho_{t} w_{y}\right\|_{L^{2}\left(\mathbf{R}^{2}\right)}$ is bounded with Cauchy-Schwartz' inequality by $\sqrt{R+c t}\left\|\rho_{t}\right\|_{L^{2}(0,2 \pi)} E_{1}^{1 / 2}$. The three other terms are bounded as explained in Appendix B:

$$
\begin{aligned}
& \left\|\frac{v}{r^{2}} \rho_{\theta \theta} w_{y}\right\|_{L^{2}\left(\mathbf{R}^{2}\right)} \leqslant c_{0} \frac{E_{6}^{1 / 2} E_{1}^{1 / 2}}{(R+c t)^{3 / 2}}, \\
& \left\|\frac{v}{r^{2}} \rho_{\theta}^{2} w_{y y}\right\|_{L^{2}\left(\mathbf{R}^{2}\right)} \leqslant c_{0} \frac{E_{1}^{1 / 2} E_{5}^{3 / 4} E_{6}^{1 / 4}}{(R+c t)^{3 / 2}} \\
& \|v(N+S)\|_{L^{2}\left(\mathbf{R}^{2}\right)} \leqslant c_{0}\left(\left(E_{1}+E_{2}\right)^{3 / 2}+\frac{E_{1}^{1 / 2}}{(R+c t)^{3 / 2}}\right) .
\end{aligned}
$$

This last inequality is also obtained by Sobolev's embedding $H^{1}\left(\mathbf{R}^{2}\right) \hookrightarrow L^{3}\left(\mathbf{R}^{2}\right)$. We then get inequality (34) for $\dot{E}_{1}$ using inequalities such as $a b \leqslant\left(a^{2}+b^{2}\right) / 2$.

Lemma 2.11. Under assumptions $(\mathrm{H})$, there exist positive constants $c_{0}, d, R_{2}, \varepsilon_{2}$ and $k>1$ such that for any $t \in(0, T]$ and $R \geqslant R_{2}, \varepsilon \leqslant \varepsilon_{2}$,

$$
\dot{E}_{2}(t) \leqslant-2 E_{3}+c_{0} E_{3}^{1 / 2}\left((d k) E_{1}^{1 / 2}+E_{1}+E_{2}+D\right) .
$$

Consequently, there exist positive constants $e_{21}, e_{22}, e_{23}, e_{25}$ and $e_{6}$ such that

$$
\begin{aligned}
\dot{E}_{2}(t) \leqslant & -2 E_{3}+\left(e_{21}+(d k)^{2}\right) E_{1}+e_{22} E_{2}+\left(e_{23}+1\right) E_{3} \\
& +e_{25} \frac{E_{5}}{(R+c t)^{2}}+\frac{e_{6}}{\sqrt{R+c t}} \frac{E_{6}}{(R+c t)^{2}}+\frac{c_{0}}{(R+c t)^{3}}
\end{aligned}
$$

where $\left\{e_{2 j}\right\}_{j=1, \ldots, 5}$ can be chosen small with appropriate $R_{2}$ and $\varepsilon_{2}$.

Proof. The proof of this lemma is very similar to the last one and we may leave it out. Notice that $k$ large enough can be chosen so that $\sup \left(F^{\prime}\left(w_{0}\right)-k \psi_{0}^{2}\right) \leqslant-2$. Then, $\sup \left|F^{\prime}\left(w_{0}\right)\right| \leqslant d k$. Once more, inequality (34) for $\dot{E}_{2}$ follows for $R \geqslant R_{2}$ and $\varepsilon \leqslant \varepsilon_{2}$. 
Lemma 2.12. Under assumptions $(\mathrm{H})$, there exist positive constants $c_{0}, d, R_{2}, \varepsilon_{2}$ such that for any $t \in(0, T]$ and any $R \geqslant R_{2}, \varepsilon \leqslant \varepsilon_{2}$,

$$
\dot{E}_{4}(t) \leqslant-d \frac{E_{5}}{(R+c t)^{2}}+c_{0} E_{4}^{1 / 2}\left(\frac{E_{5}^{3 / 4}}{(R+c t)^{3 / 2}}\left(\frac{E_{6}}{(R+c t)^{2}}\right)^{1 / 4}+A+B+G\right),
$$

where

$$
G=\frac{E_{5}^{1 / 4}}{(R+c t)^{3 / 2}}\left(\frac{E_{6}}{(R+c t)^{2}}\right)^{1 / 4}\left(E_{5}^{1 / 2}+E_{1}^{1 / 2} E_{5}^{1 / 4}\left(\frac{E_{6}}{(R+c t)^{2}}\right)^{1 / 4}+\sqrt{R+c t} E_{2}^{1 / 2}\right) .
$$

Consequently, there exist positive constants $e_{41}, e_{42}, e_{43}, e_{45}$ and $e_{46}$ such that

$$
\dot{E}_{4}(t) \leqslant-d \frac{E_{5}}{(R+c t)^{2}}+e_{41} E_{1}+e_{42} E_{2}+e_{43} E_{3}+e_{45} \frac{E_{5}}{(R+c t)^{2}}+e_{46} \frac{E_{6}}{(R+c t)^{2}}+\frac{c_{0}}{(R+c t)^{2}},
$$

where $\left\{e_{4 j}\right\}_{j=1, \ldots, 6}$ can be chosen small with appropriate $R_{2}$ and $\varepsilon_{2}$.

Proof. From Eq. (32), we bound $\dot{E}_{4}$ term by term. The only difficulty which has not been seen yet in the previous lemmas is the term $G$ which can be bounded by

$$
\int_{\Omega} \frac{\rho \rho_{\theta}}{r^{2}} \psi w_{y} \frac{\lambda_{\theta}(\infty)}{\lambda^{2}(\infty)} \mathrm{d} r \mathrm{~d} \theta
$$

with Cauchy-Schwartz's inequality.

Let us recall that $\lambda_{\theta}=\int_{0}^{\infty} \rho_{\theta}\left(\psi_{y y} v-\psi_{y} w_{y}-\psi w_{y y}\right)-\psi_{y} v_{\theta} \mathrm{d} r$. Then,

$$
\left\|\lambda_{\theta}\right\|_{L^{2}(0,2 \pi)} \leqslant c_{0}\left(\left\|\rho_{\theta}\right\|_{L^{\infty}} \frac{E_{1}^{1 / 2}}{\sqrt{R+c t}}+E_{5}^{1 / 2}+\sqrt{R+c t} E_{2}^{1 / 2}\right)
$$

and the inequality $\left\|\rho_{\theta}\right\|_{L^{\infty}} \leqslant\left(E_{5} E_{6}\right)^{1 / 4}$ ends the proof.

Lemma 2.13. Under assumptions $(\mathrm{H})$, there exist positive constants $c_{0}, R_{2}, \varepsilon_{2}$ such that for any $t \in(0, T]$ and any $R \geqslant R_{2}, \varepsilon \leqslant \varepsilon_{2}$,

$$
\dot{E}_{5}(t) \leqslant-d \frac{E_{6}}{(R+c t)^{2}}+c_{0}\left(E_{5}^{1 / 2} \frac{E_{6}}{(R+c t)^{2}}+E_{6}^{1 / 2}(A+B)\right) .
$$

Consequently, there exist positive constants $e_{51}, e_{52}, e_{53}$ and $e_{56}$ such that

$$
\begin{aligned}
\dot{E}_{5}(t) \leqslant & -d \frac{E_{6}}{(R+c t)^{2}}+e_{51} E_{1}+e_{52} E_{2}+e_{53} E_{3}+\left(e_{56}+\frac{d}{4}\right) \frac{E_{6}}{(R+c t)^{2}} \\
& +\frac{c_{0}}{(R+c t)^{2}}+\frac{2}{d}(R+c t)\left(E_{1}+E_{2}\right)^{2},
\end{aligned}
$$

where $\left\{e_{5 j}\right\}_{j=1, \ldots, 6}$ can be chosen small with appropriate $R_{2}$ and $\varepsilon_{2}$.

Proof. Once more, the proof is very similar to the previous ones, using Cauchy-Schwartz' inequality. However, we may detail how we get, from the first result, inequality (34) for $\dot{E}_{5}$. Using inequalities such as $a b \leqslant\left(a^{2}+b^{2}\right) / 2$, the only difficulties come from the terms $E_{6}^{1 / 2}\left(\left(E_{1}+E_{2}\right) / \sqrt{R+c t}\right)$ and $E_{6}^{1 / 2} /(R+c t)^{2}$ which appear in $E_{6}^{1 / 2} B$ : for any $d>0$, 


$$
\begin{aligned}
& \frac{E_{6}^{1 / 2}}{(R+c t)^{2}} \leqslant \frac{2}{d(R+c t)^{2}}+\frac{d}{8} \frac{E_{6}}{(R+c t)^{2}}, \\
& E_{6}^{1 / 2}\left(\frac{E_{1}+E_{2}}{\sqrt{R+c t}}\right) \leqslant\left(\frac{E_{6}}{(R+c t)^{2}}\right)^{1 / 2}\left(\sqrt{R+c t}\left(E_{1}+E_{2}\right)\right) \\
& \leqslant \frac{d}{8} \frac{E_{6}}{(R+c t)^{2}}+\frac{2}{d}(R+c t)\left(E_{1}+E_{2}\right)^{2} .
\end{aligned}
$$

This ends the proof of inequalities (34).

These six lemmas end the proof of Proposition 2.7 and hence of Theorem 2.5. Equipped with these energy estimates, we are able to prove the end of Theorem 2.

\subsection{Example and density of nonradial profiles}

In this paragraph, the end of Theorem 2 is proved thanks to Theorem 2.5.

Lemma 2.14. Under the assumptions of Theorem 2.5 , there exists a function $\rho_{\infty} \in L^{2}(0,2 \pi)$ such that $\rho(\cdot, t)$ converges in the $L^{2}(0,2 \pi)$ norm to $\rho_{\infty}$ as $t$ goes to infinity.

Proof. By Lemma 2.8 and Theorem 2.5, we get

$$
\left\|\rho_{t}(t)\right\|_{L^{2}(0,2 \pi)} \leqslant c_{0}\left(\frac{E_{1}+E_{2}}{\sqrt{R+c t}}+\frac{E_{3}}{R+c t}+\frac{1}{\sqrt{R+c t}} \frac{E_{6}}{(R+c t)^{2}}+\frac{1}{(R+c t)^{3 / 2}}\right) .
$$

Then, by inequality (41),

$$
\int_{0}^{t}\left\|\rho_{t}(s)\right\|_{L^{2}} \mathrm{~d} s \leqslant c_{0}\left(\frac{1+\tilde{\varepsilon}}{\sqrt{R}}\right) .
$$

As this bound is independent of $t, \int_{0}^{\infty}\left\|\rho_{t}(s)\right\|_{L^{2}(0,2 \pi)} \mathrm{d} s$ is convergent and there exists a function $\rho_{\infty} \in L^{2}(0,2 \pi)$ such that

$$
\left\|\rho_{\infty}-\rho(\cdot, t)\right\|_{L^{2}(0,2 \pi)} \leqslant \int_{t}^{\infty}\left\|\rho_{t}(s)\right\|_{L^{2}(0,2 \pi)} \mathrm{d} s
$$

converges to zero as $t$ goes to infinity. This completes the proof.

Lemma 2.15. There exist positive constants $R$ and $\varepsilon$ such that if $\tilde{u}_{0}(r, \theta)=w(r-R-\sqrt{\varepsilon /(2 \pi)} \sin \theta, r)$, the solution $u(r, \theta, t)$ of Eq. (1) with initial data $u_{0}$ converges to a non radial profile.

Proof. Take $R_{1}$ and $\varepsilon_{1}$ as in Theorem 2.5 and $R \geqslant R_{1}, \varepsilon \leqslant \varepsilon_{1}$. Then $u_{0}$ satisfies the assumptions of Theorem 2.5. Indeed, $u_{0}=w\left(r-R-\rho_{0}, r\right)+v_{0}$ where $\rho_{0}(\theta)=\sqrt{\varepsilon /(2 \pi)} \sin \theta, v_{0}=0$ and $R^{1 / 2}\left\|v_{0}\right\|_{W}^{2}+\left\|\rho_{0}\right\|_{Z}^{2}=\varepsilon$. Since $v_{0}=0$, notice that $R$ and $\varepsilon$ can be chosen independently. Therefore, choose $R$ sufficiently large so that $\sqrt{\varepsilon / 2}>c_{1}(1+\tilde{\varepsilon}) / \sqrt{R}$. Let $\tilde{u}(r, \theta, t)=w(r-s(\theta, t), r)+v(r, \theta, t)$ be the solution of Eq. (1) with initial data $u_{0}$ where $s(\theta, t)$ is defined by (9). Then, by Lemma 2.14, $\int_{0}^{t}\left\|\rho_{t}\right\|_{L^{2}(0,2 \pi)} \mathrm{d} s \leqslant c_{0}(1+\tilde{\varepsilon}) \sqrt{R}$. Finally,

$$
\left\|\rho(\theta, t)-\sqrt{\frac{\varepsilon}{2 \pi}} \sin \theta\right\|_{L^{2}(0,2 \pi)} \leqslant c_{0}\left(\frac{1+\tilde{\varepsilon}}{\sqrt{R}}\right)
$$


for any $t \geqslant 0$. If there exists some $t>0$ such that $\rho(\theta, t)=\rho$ is independent of $\theta$, then

$$
\left\|\rho-\sqrt{\frac{\varepsilon}{2 \pi}} \sin \theta\right\|_{L^{2}(0,2 \pi)}=\sqrt{2 \pi \rho^{2}+\frac{\varepsilon}{2}}>c_{1}\left(\frac{1+\tilde{\varepsilon}}{\sqrt{R}}\right) .
$$

This contradicts the latter inequality. Therefore, for any $t \geqslant 0, \rho(\theta, t)$ is not constant.

Moreover, as Theorem 2.5 is satisfied, $\|v\|_{W}$ converges to zero as $t$ goes to infinity and $u(r, \theta, t)$ converges to a nonradial profile as $t$ goes to infinity.

This ends the proof of Theorem 2. We give a few more information by introducing two new spaces as follows:

$$
\begin{aligned}
\mathcal{S}_{1}=\left\{u_{0} \in H^{1}\left(\mathbf{R}^{2}\right) \mid\right. & \text { for some } R \geqslant \max \left(R_{1}, R_{1}^{\prime}\right), \tilde{u}_{0}(r, \theta)-w(r-R, r) \equiv \\
& \left.\xi(r, \theta) \text { satisfies Lemma } 2.3 \text { and }\left(v_{0}, \rho_{0}\right) \in W \times Z \text { satisfy Theorem } 2.5\right\} .
\end{aligned}
$$

Moreover, there exists, for any function $u_{0} \in \mathcal{S}_{1}$, a unique function $\rho_{\infty} \in L^{2}(0,2 \pi)$ satisfying Lemma 2.14. We call $\mathcal{S}_{2}$ the set of all these functions $\rho_{\infty} \in L^{2}(0,2 \pi)$ satisfying the above properties for $u_{0} \in \mathcal{S}_{1}$.

Lemma 2.16. $\mathcal{S}_{2}$ is a subset of $L^{2}(0,2 \pi)$ which contains some non constant functions and $\mathcal{S}_{2}$ is dense in the ball $B\left(0, \min \left(\delta_{1}^{\prime}, \sqrt{\varepsilon_{1}}\right)\right)$ of $Z$.

Proof. For any $\rho_{\infty} \in \mathcal{S}_{2}$, we know that $\rho_{\infty} \in L^{2}(0,2 \pi)$. Moreover, there exists, by Lemma 2.15 , some $u_{0} \in \mathcal{S}_{1}$ such that $\rho_{\infty} \in \mathcal{S}_{2}$ is not constant.

Take now $\rho \in B\left(0, \min \left(\delta_{1}^{\prime}, \sqrt{\varepsilon_{1}}\right)\right)$ and $R \geqslant \max \left(R_{1}, R_{1}^{\prime}\right)$. Define $\tilde{u}_{0} \in H^{1}\left(\mathbf{R}^{2}\right)$ by $u_{0}(r, \theta)=w(r-R-$ $\rho(\theta), r)$. Then, $\left\|u_{0}-w(r-R, r)\right\|_{W} \leqslant\|\rho\|_{Z} \leqslant \delta_{1}^{\prime}$, and by Lemma 2.3, there exists a unique pair $\left(v_{0}, \rho_{0}\right) \in W \times Z$ satisfying

$$
\begin{aligned}
& \tilde{u}_{0}(r, \theta)=w\left(r-R-\rho_{0}(\theta), r\right)+v_{0}(r, \theta), \\
& \left\langle v_{0}, \psi\right\rangle=0, \\
& \left\|v_{0}\right\|_{W}+\left\|\rho_{0}\right\|_{Z} \leqslant K^{\prime} \min \left(\delta_{1}^{\prime}, \sqrt{\varepsilon_{1}}\right) .
\end{aligned}
$$

As a consequence, $\rho_{0} \equiv \rho$ and $v_{0} \equiv 0$ and

$$
R^{1 / 2}\left\|v_{0}\right\|_{W}^{2}+\left\|\rho_{0}\right\|_{Z}^{2}=\|\rho\|_{Z}^{2} \leqslant \varepsilon_{1} \text {. }
$$

Notice that as $v_{0}=0$, this last inequality is still valid for arbitrary large $R$. Finally by Theorem 2.5 and Lemma 2.14 , there exist $\left(v, \rho, \rho_{\infty}\right) \in C\left(\mathbf{R}^{+}, W \times Z\right) \times L^{2}(0,2 \pi)$ such that

$$
\begin{aligned}
& (R+c t)^{1 / 2}\|v\|_{W}^{2}+\|\rho\|_{Z}^{2} \leqslant n\left(\varepsilon_{1}+\frac{1}{R}\right) \\
& \lim _{t \rightarrow+\infty}\left\|\rho(\cdot, t)-\rho_{\infty}\right\|_{L^{2}(0,2 \pi)}=0, \\
& \left\|\rho_{0}-\rho_{\infty}\right\|_{L^{2}(0,2 \pi)} \leqslant \frac{c_{1}}{\sqrt{R}} .
\end{aligned}
$$

As $R$ can be chosen as large as we need it, the last inequality shows that $\mathcal{S}_{2}$ is dense in $B_{Z}\left(0, \min \left(\delta_{1}^{\prime}, \sqrt{\varepsilon_{1}}\right)\right)$.

\section{Appendix A. Perturbation theorem for evolution operators}

Theorem A.1. Let $A$ be a sectorial operator on a Banach space $X$ such that $\operatorname{Re}(\sigma(A)) \geqslant a>0$ and $\alpha \in[0,1)$. We set $X^{\alpha} \equiv D\left(A^{\alpha}\right)$. Let $\beta>0, M>0$ so that

$$
\left\|\mathrm{e}^{-t A}\right\|_{\mathcal{L}(X)} \leqslant M \mathrm{e}^{-\beta t} \text { and }\left\|\mathrm{e}^{-t A} x\right\|_{X^{\alpha}} \leqslant \frac{M}{t^{\alpha}} \mathrm{e}^{-\beta t}\|x\|_{X}
$$


for all $t>0$ and $x \in X$. Suppose $B:\left[t_{0} ;+\infty\right) \rightarrow \mathcal{L}\left(X^{\alpha}, X\right)$ is locally Hölder continuous with

$$
\|B(t)\|_{\mathcal{L}\left(X^{\alpha}, X\right)} \leqslant \gamma
$$

for all $t \geqslant t_{0} \geqslant 0$ and some $\gamma>0$. Let $T(t, \tau), t_{0} \leqslant \tau \leqslant t$, be the family of evolution operators so that the unique solution of

$$
\begin{aligned}
& \frac{\mathrm{d} x}{\mathrm{~d} t}+A x=B(t) x, \quad t \geqslant \tau, \\
& x(\tau)=x_{0},
\end{aligned}
$$

is $x\left(t ; \tau, x_{0}\right)=T(t, \tau) x_{0}, t_{0} \leqslant \tau \leqslant t$. Then, there exists $\gamma_{0}>0$ such that for any $\gamma \in\left(0, \gamma_{0}\right)$, there exists $\delta \in(0, \beta)$ such that for any $t_{0} \leqslant s \leqslant t$,

$$
\|T(t, s)\|_{\mathcal{L}(X)} \leqslant M_{1} \mathrm{e}^{-\delta(t-s)} .
$$

Proof. Given $x_{0} \in X, t_{0} \leqslant \tau \leqslant T$ and $\delta \in(0, \beta)$, we shall solve (46) in the Banach space

$$
V=\left\{x \in C^{0}([\tau, T], X) \cap C^{0}\left((\tau, T], X^{\alpha}\right) \mid\|x\|_{V}<\infty\right\},
$$

where

$$
\|x\|_{V}=\sup _{\tau \leqslant t \leqslant T} \mathrm{e}^{\delta(t-\tau)}\|x(t)\|_{X}+\sup _{\tau<t \leqslant T}(t-\tau)^{\alpha} \mathrm{e}^{\delta(t-\tau)}\|x(t)\|_{X^{\alpha}} .
$$

First, given $x \in V$, we define the function $F$ from $V$ to $V$ by

$$
F(x)(t)=\mathrm{e}^{-A(t-\tau)} x_{0}+\int_{\tau}^{t} \mathrm{e}^{-A(t-s)} B(s) x(s) \mathrm{d} s .
$$

For $r>0$, let $\gamma_{0}>0$ and $R>0$ be chosen so that

$$
\begin{aligned}
& c(T)=\sup _{\tau \leqslant t \leqslant T} \int_{\tau}^{t} \frac{\mathrm{d} s}{(t-s)^{\alpha}(s-\tau)^{\alpha}}, \quad R=4 M r, \\
& C_{1}=M \gamma_{0} \mathrm{e}^{(\delta-\beta)(T-\tau)}(T-\tau)^{\alpha} c(T) \leqslant \frac{1}{4}, \quad C_{2}=M \gamma_{0} \mathrm{e}^{-\delta(T-\tau)} \frac{(T-\tau)^{1-\alpha}}{1-\alpha} \leqslant \frac{1}{4} .
\end{aligned}
$$

Then, for any $x_{0} \in X$ with $\left\|x_{0}\right\|_{X} \leqslant r, F$ maps the ball $B_{V}(0, R)$ of $V$ into itself and has a unique fixed point in the ball $B_{V}(0, R)$. Using Gronwall's lemma, it is then straightforward to show that this fixed point is actually the unique solution of (46) in the space $V$. Finally, since $\|x\|_{V} \leqslant 2 M\left\|x_{0}\right\|_{X}+\left(C_{1}+C_{2}\right)\|x\|_{V}$, the solution $x(t)$ is defined for all $t>0$ and the bound (47) holds with $M_{1}=4 M$.

\section{Appendix B. A few lemmas}

\section{B.1. Schur's lemma}

Lemma B.1. Let $P$ be an operator of $L^{2}\left(\mathbf{R}^{2}\right)$ defined in polar coordinates by

$$
P u(r, \theta)=\int_{0}^{\infty} u(z, \theta) K(z, r, \theta) d z, \quad u \in L^{2}\left(\mathbf{R}^{2}\right)
$$

so that 


$$
\begin{aligned}
& c_{1}=\sup _{z \geqslant 0, \theta \in[0,2 \pi)} \int_{0}^{\infty}|K(z, r, \theta)| \sqrt{\frac{r}{z}} \mathrm{~d} r<\infty, \\
& c_{2}=\sup _{r \geqslant 0, \theta \in[0,2 \pi)} \int_{0}^{\infty}|K(z, r, \theta)| \sqrt{\frac{r}{z}} \mathrm{~d} z<\infty .
\end{aligned}
$$

Then, $P$ is continuous on $L^{2}\left(\mathbf{R}^{2}\right)$ and for any $u \in L^{2}\left(\mathbf{R}^{2}\right)$,

$$
\|P u\|_{L^{2}\left(\mathbf{R}^{2}\right)} \leqslant \sqrt{c_{1} c_{2}}\|u\|_{L^{2}\left(\mathbf{R}^{2}\right)} .
$$

Proof. We fix $\theta \in[0,2 \pi)$. Then, by Hölder's inequality and Fubini's theorem,

$$
\begin{aligned}
\int_{0}^{\infty}\left[\int_{0}^{\infty} K(z, r, \theta) u(z, \theta) \mathrm{d} z\right]^{2} r \mathrm{~d} r & \leqslant \int_{0}^{\infty}\left(\int_{0}^{\infty} K \frac{\mathrm{d} z}{\sqrt{z}}\right)\left(\int_{0}^{\infty} K u^{2} \sqrt{z} \mathrm{~d} z\right) r \mathrm{~d} r \\
& \leqslant c_{2} \int_{0}^{\infty} u^{2}(z) z \int_{0}^{\infty} K \sqrt{\frac{r}{z}} \mathrm{~d} r \mathrm{~d} z \leqslant c_{1} c_{2} \int_{0}^{\infty} u^{2}(z, \theta) z \mathrm{~d} z
\end{aligned}
$$

Integrating in $\theta \in(0,2 \pi)$ the above inequality, we get the continuity of $P$.

Throughout the proof of Lemma 2.7, we use Schur's lemma in the following way, most of the time without mentioning it. For instance, the following inequality

$$
\int_{0}^{2 \pi} \int_{0}^{R+c t}\left(\int_{0}^{r} \psi v_{\theta} \mathrm{d} z\right)^{2} r \mathrm{~d} r \mathrm{~d} \theta \leqslant c_{0}(R+c t)^{2} \int_{0}^{2 \pi} \int_{0}^{\infty} \frac{v_{\theta}^{2}}{r^{2}} r \mathrm{~d} r \mathrm{~d} \theta
$$

is proved by Schur's lemma by writing

$$
K(z, r, \theta, t)=\mathbb{I}_{z \leqslant r \leqslant(R+c t)} \psi(z-s(\theta, t), z) z \quad \text { and } \quad u(z, \theta, t)=\frac{v_{\theta}}{z} .
$$

Then, $c_{i}(t) \leqslant c_{0}(R+c t)$ for $i=1,2$. This concludes the proof of the above inequality.

\section{B.2. Jensen's inequality}

Proposition B.2. Let $\phi$ be a convex function and $v$ a probability measure on a measurable set $A$. Then, for any $f \in L^{1}(A, \mathrm{~d} v)$,

$$
\phi\left(\int_{A} f \mathrm{~d} v\right) \leqslant \int_{A} \phi(f) \mathrm{d} v .
$$

\section{Corollary B.3.}

$$
\int_{0}^{2 \pi}\left(\int_{0}^{\infty} v(r, \theta, t) \psi(r-s(\theta, t), r) \mathrm{d} r\right)^{2} \mathrm{~d} \theta \leqslant c_{0} \int_{0}^{2 \pi} \int_{0}^{\infty} v^{2} \psi(r-s(\theta, t), r) \mathrm{d} r \mathrm{~d} \theta .
$$


Proof. For any $\theta \in(0,2 \pi)$ and any $t>0$, let $\mathrm{d} \nu=\tilde{\alpha} \psi(r-s(\theta, t), r) \mathrm{d} r$ where $\tilde{\alpha}$ is chosen so that $\int_{\mathbf{R}} \tilde{\alpha} \psi(r-$ $s(\theta, t), r) \mathrm{d} r=1$. Then, $v$ is a probability measure for any fixed $t$ and $\theta$, and $\phi(x)=x^{2}$ is convex in $\mathbf{R}^{2}$. By Jensen's inequality,

$$
\int_{0}^{2 \pi}\left(\int_{0}^{\infty} v(r, \theta, t) \psi(r-s(\theta, t), r) \mathrm{d} r\right)^{2} \mathrm{~d} \theta \leqslant \int_{0}^{2 \pi} \int_{0}^{\infty} \frac{1}{\tilde{\alpha}} v^{2} \psi(r-s(\theta, t), r) \mathrm{d} r \mathrm{~d} \theta .
$$

As $\tilde{\alpha}^{-1}$ can be bounded independently of $\theta$ and $t$, this ends the proof.

\section{Appendix C. Log-concave functions}

Proposition B.1. Let $F \in C^{3}(\mathbf{R})$ be a function satisfying the following conditions:

$$
\begin{aligned}
& F(0)=F(1)=0, \quad F^{\prime}(0)=\alpha<0, \quad F^{\prime}(1)=\beta<0, \\
& \exists \mu \in(0,1) \text { so that } F(u)>0 \text { for } u \in(\mu, 1), \quad F(u)<0 \text { for } u \in(0, \mu), \\
& \int_{0}^{1} F(u) \mathrm{d} u>0, \quad F^{(3)}(u) \leqslant 0 \quad \text { for all } u \in[0,1] .
\end{aligned}
$$

Let $c>0$ and $w_{0} \in C^{2}(\mathbf{R})$ be a monotone solution of the $O D E$

$$
w_{0}^{\prime \prime}+c w_{0}^{\prime}+F\left(w_{0}\right)=0, \quad x \in \mathbf{R},
$$

with the boundary conditions at infinity

$$
\lim _{x \rightarrow-\infty} w_{0}(x)=1 \text { and } \lim _{x \rightarrow+\infty} w_{0}(x)=0 .
$$

Define $\phi_{0}=w_{0}^{\prime}<0$. Then, $\phi_{0}$ is log-concave:

$$
-\left(\frac{\phi_{0}^{\prime}}{\phi_{0}}\right)^{\prime}>0 \text {. }
$$

Proof. As $-\phi_{0}^{\prime} / \phi_{0}=-w_{0}^{\prime \prime} / w_{0}^{\prime}=c+F\left(w_{0}\right) / w_{0}^{\prime} \equiv c+g$, it is sufficient to prove that $g$ is increasing on $\mathbf{R}$, i.e., that $h \equiv g^{\prime}$ is positive. We first study the behaviour of $g$ and $h$ as $|x|$ goes to infinity. It is a standard result that $w_{0}$ (resp. $1-w_{0}$ ) decreases exponentially fast to zero as $x$ goes to $+\infty$ (resp. $-\infty$ ). Let us begin with the behaviour of $w_{0}$ at $-\infty$ :

$$
w_{0}(x)=1-\mathrm{e}^{\lambda x}+A \mathrm{e}^{2 \lambda x}+\mathrm{o}\left(\mathrm{e}^{2 \lambda x}\right),
$$

where $\lambda>0$. Then,

$$
\begin{aligned}
& w_{0}^{\prime}(x)=-\lambda \mathrm{e}^{\lambda x}+2 \lambda A \mathrm{e}^{2 \lambda x}+\mathrm{o}\left(\mathrm{e}^{2 \lambda x}\right), \\
& w_{0}^{\prime \prime}(x)=-\lambda^{2} \mathrm{e}^{\lambda x}+4 \lambda^{2} A \mathrm{e}^{2 \lambda x}+\mathrm{o}\left(\mathrm{e}^{2 \lambda x}\right),
\end{aligned}
$$

and by Taylor's theorem,

$$
\begin{aligned}
F\left(w_{0}(x)\right) & =F^{\prime}(1)\left(w_{0}(x)-1\right)+\frac{1}{2} F^{\prime \prime}(1)\left(w_{0}(x)-1\right)^{2}+\mathrm{o}\left(\mathrm{e}^{2 \lambda x}\right) \\
& =\beta\left(-\mathrm{e}^{\lambda x}+A \mathrm{e}^{2 \lambda x}\right)+\frac{1}{2} F^{\prime \prime}(1) \mathrm{e}^{2 \lambda x}+\mathrm{o}\left(\mathrm{e}^{2 \lambda x}\right) .
\end{aligned}
$$


As $w_{0}$ is a solution of (48), the first order of the expansion says that $\lambda$ is the positive root of

$$
\lambda^{2}+c \lambda+\beta=0 \text {. }
$$

The second order gives

$$
A\left(4 \lambda^{2}+2 c \lambda+\beta\right)+\frac{1}{2} F^{\prime \prime}(1)=0
$$

i.e., $A\left(3 \lambda^{2}+c \lambda\right)+\frac{1}{2} F^{\prime \prime}(1)=0$. Notice that the above assumptions on $F$ forces $F^{\prime \prime}(1)$ to be negative. Therefore, $A>0$. Finally,

$$
g=-\frac{w_{0}^{\prime \prime}}{w_{0}^{\prime}}-c=-(c+\lambda)+2 A \lambda \mathrm{e}^{\lambda x}+\mathrm{o}\left(\mathrm{e}^{2 \lambda x}\right)
$$

and $h \sim 2 A \lambda^{2} \mathrm{e}^{\lambda x}$ as $x$ goes to $-\infty$. We can then conclude from this study that $h$ is positive for $x<0$ sufficiently large.

A similar study in $+\infty$ with $w_{0}(x)=\mathrm{e}^{\mu x}-B \mathrm{e}^{2 \mu x}+\mathrm{o}\left(\mathrm{e}^{2 \mu x}\right)$ where $\mu$ is the negative root of $\mu^{2}+c \mu+\alpha=0$, gives that $-B\left(2 \mu^{2}-\alpha\right)+\frac{1}{2} F^{\prime \prime}(0)=0$ which implies that $B>0$. Finally, as $g(x)=-(c+\mu)+2 B \mu \mathrm{e}^{\mu x}+\mathrm{o}\left(\mathrm{e}^{2 \mu x}\right)$,

$$
h(x) \sim 2 B \mu^{2} \mathrm{e}^{\mu x} \quad \text { when } x \rightarrow+\infty
$$

and $h$ is positive for $x>0$ sufficiently large.

Suppose now that there exists some $x_{0} \in \mathbf{R}$ such that $h\left(x_{0}\right) \leqslant 0$ and define

$$
x_{1}=\inf \{x \in \mathbf{R} \mid h(x) \leqslant 0\}, \quad x_{2}=\sup \{x \in \mathbf{R} \mid h(x) \leqslant 0\} .
$$

Then, $h^{\prime}\left(x_{1}\right) \leqslant 0$ and $h^{\prime}\left(x_{2}\right) \geqslant 0$. As $h=c g+g^{2}+F^{\prime}\left(w_{0}\right)$, we get

$$
h^{\prime}=c(1+2 g) h+F^{\prime \prime}\left(w_{0}\right) w_{0}^{\prime} .
$$

Then, $F^{\prime \prime}\left(w_{0}\left(x_{1}\right)\right) \geqslant 0$ and $F^{\prime \prime}\left(w_{0}\left(x_{2}\right)\right) \leqslant 0$. As $x_{1} \leqslant x_{2}$ and $F^{\prime \prime}\left(w_{0}\right)$ is increasing, we conclude that

$$
F^{\prime \prime}\left(w_{0}(x)\right)=0 \quad \text { for all } x \in\left[x_{1}, x_{2}\right] \text {. }
$$

Then, $F^{\prime \prime}\left(w_{0}(x)\right) \geqslant 0$ for all $x \geqslant x_{2}$ and by (49),

$$
\left\{\begin{array}{l}
h^{\prime}(x) \leqslant c(1+2 g(x)) h(x), \quad x \in\left[x_{2},+\infty\right), \\
h\left(x_{2}\right)=0 .
\end{array}\right.
$$

Finally, by the maximum principle, $h(x) \leqslant 0$ for all $x \geqslant x_{2}$ which contradicts the definition of $x_{2}$. Therefore, $h$ is positive on $\mathbf{R}$ and $g$ is increasing. This concludes the proof.

\section{Acknowledgements}

I would like to thank Professor Thierry Gallay for all his help and suggestions regarding this work.

\section{References}

[1] D.G. Aronson, H.F. Weinberger, Nonlinear diffusion in population genetics, combustion, and nerve propagation, in: Partial Differential Equations and Related Topics, in: Lecture Notes in Math., vol. 446, Springer, New York, 1975, pp. 5-49.

[2] D.G. Aronson, H.F. Weinberger, Multidimensional nonlinear diffusion arising in population genetics, Adv. Math. 30 (1978) $33-76$.

[3] P.C. Fife, Long time behaviour of solutions of bistable nonlinear diffusion equations, Arch. Rational Mech. Anal. 70 (1979) 31-46.

[4] P.C. Fife, J.B. McLeod, The approach of solutions of nonlinear diffusion equations to travelling front solutions, Arch. Rational Mech. Anal. 65 (1977) 335-361. 
[5] R.A. Fisher, The advance of advantageous genes, Ann. of Eugenics 7 (1937) 355-369.

[6] D. Henry, Geometric Theory of Semilinear Parabolic Equations, in: Lecture Notes in Math., vol. 840, Springer-Verlag, New York, 1981.

[7] C.K.R.T. Jones, Spherically symmetric solutions of a reaction-diffusion equation, J. Differential Equations 49 (1983) $142-169$.

[8] C.K.R.T. Jones, Asymptotic behaviour of a reaction-diffusion equation in higher space dimensions, Rocky Mountain J. Math. 13 (1983) 355-364.

[9] Ya.I. Kanel', On the stability of solutions of the cauchy problem for equations arising in the theory of combustion, Mat. Sb. 59 (1962) 245-288.

[10] T. Kapitula, Multidimensional stability of planar travelling waves, Trans. Amer. Math. Soc. 349 (1997) $257-269$.

[11] A.N. Kolmogorov, I.G. Petrovskii, N.S. Piskunov, A study of the equation of diffusion with increase in the quantity of matter, and its application to a biological problem, Bull. Moskovsk. Gos. Univ. 1 (7) (1937) 1-26.

[12] C.D. Levermore, J.X. Xin, Multidimensional stability of travelling waves in a bistable reaction-diffusion equation II, Comm. Partial Differential Equations 17 (1992) 1901-1924.

[13] A. Pazy, Semigroups of Linear Operators and Applications to Partial Differential Equations, Springer-Verlag, New York, 1983.

[14] D.H. Sattinger, Weighted norms for the stability of travelling waves, J. Differential Equations 25 (1977) $130-144$.

[15] A.E. Taylor, Introduction to Functional Analysis, Wiley, New York, 1961.

[16] K. Uchiyama, Asymptotic behaviour of solutions of reaction-diffusion equations with varying drift coefficients, Arch. Rational Mech. Anal. 90 (1983) 291-311.

[17] J.X. Xin, Multidimensional stability of travelling waves in a bistable reaction-diffusion equation I, Comm. Partial Differential Equations 17 (1992) 1889-1899. 